\title{
LIMIT THEOREMS FOR A CLASS OF CRITICAL SUPERPROCESSES WITH STABLE BRANCHING
}

\author{
YAN-XIA REN, RENMING SONG AND ZHENYAO SUN
}

\begin{abstract}
We consider a critical superprocess $\left\{X ; \mathbf{P}_{\mu}\right\}$ with general spatial motion and spatially dependent stable branching mechanism with lowest stable index $\gamma_{0}>$ 1. We first show that, under some conditions, $\mathbf{P}_{\mu}\left(\left\|X_{t}\right\| \neq 0\right)$ converges to 0 as $t \rightarrow$ $\infty$ and is regularly varying with index $\left(\gamma_{0}-1\right)^{-1}$. Then we show that, for a large class of non-negative testing functions $f$, the distribution of $\left\{X_{t}(f) ; \mathbf{P}_{\mu}\left(\cdot\left\|X_{t}\right\| \neq 0\right)\right\}$, after appropriate rescaling, converges weakly to a positive random variable $\mathbf{z}^{\left(\gamma_{0}-1\right)}$ with Laplace transform $E\left[e^{-u \mathbf{z}^{\left(\gamma_{0}-1\right)}}\right]=1-\left(1+u^{-\left(\gamma_{0}-1\right)}\right)^{-1 /\left(\gamma_{0}-1\right)}$.
\end{abstract}

\section{INTRODUCTION}

1.1. Background. The study of the asymptotic behaviors of critical branching particle systems has a long history. It is well known that for a critical Galton-Watson process $\left\{\left(Z_{n}\right)_{n \geq 0} ; P\right\}$, we have

$$
n P\left(Z_{n}>0\right) \underset{n \rightarrow \infty}{\longrightarrow} \frac{2}{\sigma^{2}}
$$

and

$$
\left\{\frac{Z_{n}}{n} ; P\left(\cdot \mid Z_{n}>0\right)\right\} \underset{n \rightarrow \infty}{\stackrel{\text { law }}{\longrightarrow}} \frac{\sigma^{2}}{2} \mathbf{e},
$$

where $\sigma^{2}$ is the variance of the offspring distribution and $\mathbf{e}$ is an exponential random variable with mean 1. The result (1.1) is due to Kolmogorov [17], and the result (1.2) is due to Yaglom [33]. For further references to these results, see [10, 13]. Since then, lots of analogous results have been obtained for more general critical branching processes with finite 2nd moment, see [1, 2, 3, 12, for example.

Notice that (1.1) and (1.2) are still valid when $\sigma^{2}=\infty$, see [13] for example. In this case, the limits in (1.1) and (1.2) are degenerate, and thus more appropriate scalings are needed. Research in this direction was first conducted by Zolotarev [34] in a simplified continuous time set-up, which is then extended by Slack [30] to discrete time critical

\footnotetext{
2010 Mathematics Subject Classification. Primary: 60J68; 60F05. Secondary 60J80; 60J25.

Key words and phrases. Critical superprocess; stable branching, scaling limit; intrinsic ultracontractivity; regular variation.

The research of Yan-Xia Ren is supported in part by NSFC (Grant Nos. 11671017 and 11731009), and LMEQF. .

The research of Renming Song is supported in part by the Simons Foundation (\#429343, Renming

Zhenyao Sun is the corresponding author.
} Song). 
Galton-Watson processes allowing infinite variance. The main result of [30] can be stated as follows. Consider a critical Galton-Watson process $\left\{\left(Z_{n}\right)_{n \geq 0} ; P\right\}$. Assume that the generating function $f(s)$ of the offspring distribution is of the form

$$
f(s)=s+(1-s)^{1+\alpha} l(1-s), \quad s \geq 0,
$$

where $\alpha \in(0,1]$ and $l$ is a function slowly varying at 0 . Then

$$
P\left(Z_{n}>0\right)=n^{-1 / \alpha} L(n),
$$

where $L$ is a function slowly varying at $\infty$, and

$$
\left\{P\left(Z_{n}>0\right) Z_{n} ; P\left(\cdot \mid Z_{n}>0\right)\right\} \underset{n \rightarrow \infty}{\stackrel{\text { law }}{\longrightarrow}} \mathbf{z}^{(\alpha)},
$$

where $\mathbf{z}^{(\alpha)}$ is a positive random variable with Laplace transform

$$
E\left[e^{-u \mathbf{z}^{(\alpha)}}\right]=1-\left(1+u^{-\alpha}\right)^{-1 / \alpha}, \quad u \geq 0 .
$$

In 31, Slack also considered the converse of this problem: In order for $\left\{P\left(Z_{n}>\right.\right.$ 0) $\left.Z_{n} ; P\left(\cdot \mid Z_{n}>0\right)\right\}$ to have a non-degenerate weak limit, the generating function of the offspring distribution must be of the form of (1.3) for some $0<\alpha \leq 1$. For shorter and more unified approaches to these results, we refer our readers to [5, 22].

Goldstein and Hoppe [9] considered the asymptotic behavior of multitype critical Galton-Watson processes without the 2nd moment condition. Their main result can be stated as follows. Let $\mathbf{Z}_{n}=\left(Z_{n}^{(1)}, \ldots, Z_{n}^{(d)}\right)$ be a $d$-type, nonsingular Galton-Watson process with its mean matrix $M:=\left(E\left[Z_{1}^{(j)} \mid Z_{0}^{(i)}=1, Z_{0}^{(k)}=0, \forall k \neq i\right]\right)_{1 \leq i, j \leq d}$ being positive regular, that is, all entries of $M$ are finite and there exists a number $n \geq 1$ such that all entries of $M^{n}$ are positive. Denote by $\mathbf{F}(\mathbf{s})=\left(\mathbf{F}_{1}(\mathbf{s}), \ldots, \mathbf{F}_{d}(\mathbf{s})\right)$ the generating function of the offspring distribution, and by $\mathbf{F}^{(n)}(\mathbf{s}), n>1$, its $n$th iterates. Assume that the process is critical in the sense that the maximal eigenvalue of $M$ is 1 . Let $\mathbf{v}$ and $\mathbf{u}$ be the left and right eigenvectors of $M$, respectively, corresponding to this maximal eigenvalue 1 , and normalized so that $\mathbf{v} \cdot \mathbf{u}=1$ and $\mathbf{1} \cdot \mathbf{u}=1$, where $\mathbf{1}$ is the vector $(1, \ldots, 1)$. Suppose that

$$
\mathbf{v} G(\mathbf{1}-x \mathbf{u}) \mathbf{u}=x^{\alpha} l(x), \quad x>0,
$$

where $0<\alpha \leq 1 ; l$ is slowly varying at 0 ; and the matrix $G(\mathbf{s})$ is defined by

$$
\mathbf{1}-\mathbf{F}(\mathbf{s})=(M-G(\mathbf{s}))(\mathbf{1}-\mathbf{s}), \quad \mathbf{s} \in \mathbb{R}_{+}^{d} .
$$

Let $a_{n}:=\mathbf{v} \cdot\left(\mathbf{1}-\mathbf{F}^{(n)}(\mathbf{0})\right)$, with $\mathbf{0} \in \mathbb{R}_{+}^{d}$ being the vector $(0, \ldots, 0)$. It was shown in [9] that, for each $\mathbf{i} \in \mathbb{N}_{0}^{d} \backslash\{\mathbf{0}\}$,

$$
n l\left(a_{n}\right) \mathrm{P}\left(\mathbf{Z}_{n} \neq \mathbf{0} \mid Z_{0}=\mathbf{i}\right)^{\alpha} \underset{n \rightarrow \infty}{\longrightarrow} \frac{(\mathbf{i} \cdot \mathbf{u})^{\alpha}}{\alpha},
$$

and for each $\mathbf{j} \in \mathbb{N}_{0}^{d}$,

$$
\left\{a_{n} \mathbf{Z}_{n} \cdot \mathbf{j} ; P\left(\cdot \mid \mathbf{Z}_{n} \neq \mathbf{0}, \mathbf{Z}_{0}=\mathbf{i}\right)\right\} \underset{n \rightarrow \infty}{\stackrel{\text { law }}{\longrightarrow}}(\mathbf{v} \cdot \mathbf{j}) \mathbf{z}^{(\alpha)},
$$

where $\mathbf{z}^{(\alpha)}$ is a random variable with Laplace transform given by (1.6). For the converse of this problem, Vatutin [32] showed that in order for the left side of (1.9) to have a 
non-degenerate weak limit, one must have (1.7) for some $0<\alpha \leq 1$. Vatutin [32] also considered analogous results for continuous time multitype critical Galton-Watson processes.

Asmussen and Hering [1, Sections 6.3 and 6.4] discussed similar questions for critical branching Markov processes $\left(Y_{t}\right)$ in a general space $E$ under some ergodicity condition (the so-called condition (M), see [1, p. 156]) on the mean semigroup of $\left(Y_{t}\right)$. When the second moment is infinite, under a condition parallel to (1.7) (the so-called condition (S) [1, p. 207]), results parallel to (1.8) and (1.9) were proved in [1, Theorem 6.4.2] for critical branching Markov processes.

In this paper, we are interested in a class of measure-valued branching Markov processes known as $(\xi, \psi)$-superprocesses: $\xi$, the spatial motion of the superprocess, is a Hunt process on a locally compact separable metric space $E$; $\psi$, the branching mechanism of the superprocess, is a function on $E \times[0, \infty)$ of the form

$$
(1.10) \psi(x, z):=-\beta(x) z+\sigma(x)^{2} z^{2}+\int_{(0, \infty)}\left(e^{-z y}-1+z y\right) \pi(x, d y), \quad x \in E, z \geq 0,
$$

where $\beta, \sigma \in \mathscr{B}_{b}(E)$ and $\pi(x, d y)$ is a kernel from $E$ to $(0, \infty)$ such that $\sup _{x \in E} \int_{(0, \infty)}(y \wedge$ $\left.y^{2}\right) \pi(x, d y)<\infty$. For the precise definition and properties of superprocesses, see [20].

Results parallel to (1.1) and (1.2) have been obtained for some critical superprocesses by Evans and Perkins [8] and Ren, Song and Zhang [26]. Evans and Perkins [8] considered critical superprocesses with branching mechanism of the form $(x, z) \mapsto z^{2}$ and with the spatial motion satisfying some ergodicity conditions. Ren, Song and Zhang [26] extended the results of [8] to a class of critical superprocesses with general branching mechanism and general spatial motions. The main results of [26] are as follows. Let $\left\{\left(X_{t}\right)_{t \geq 0} ; \mathbf{P}_{\mu}\right\}$ be a critical superprocess starting from a finite measure $\mu$ on $E$. Suppose the spatial motion $\xi$ is intrinsically ultracontractive with respect to some reference measure $m$, and the branching mechanism $\psi$ satisfies the following second moment condition

$$
\sup _{x \in E} \int_{(0, \infty)} y^{2} \pi(x, d y)<\infty .
$$

For any finite measure $\mu$ on $E$ and any measurable function $f$ on $E$, we use $\langle f, \mu\rangle$ to denote the integral of $f$ with respect to $\mu$. Put $\|\mu\|=\langle 1, \mu\rangle$. Under some other mild assumptions, it was proved in [26] that

$$
t \mathbf{P}_{\mu}\left(\left\|X_{t}\right\| \neq 0\right) \underset{t \rightarrow \infty}{\longrightarrow} c^{-1}\langle\phi, \mu\rangle,
$$

and for a large class of testing functions $f$ on $E$,

$$
\left\{t^{-1} X_{t}(f) ; \mathbf{P}_{\mu}\left(\cdot \mid\left\|X_{t}\right\| \neq 0\right)\right\} \underset{t \rightarrow \infty}{\stackrel{\text { law }}{\longrightarrow}} c\left\langle\phi^{*}, f\right\rangle_{m} \mathbf{e} .
$$

Here, the constant $c>0$ is independent of the choice of $\mu$ and $f ;\langle\cdot, \cdot\rangle_{m}$ denotes the inner product in $L^{2}(E, m)$; e is an exponential random variable with mean 1 ; and $\phi$ (respectively, $\phi^{*}$ ) is the principal eigenfunction of (respectively, the dual of) the generator of the mean semigroup of $X$. In [25], we provided an alternative probabilistic approach to (1.12) and (1.13). 
It is natural to ask whether results parallel to (1.4) and (1.5) are still valid for some critical superprocesses without the second moment condition (1.11). A simpler version of this question has already been answered in the context of continuous-state branching processes (CSBPs) which can be viewed as superprocesses without spatial movements. Kyprianou and Pardo [19] considered CSBPs $\left\{\left(Y_{t}\right)_{t \geq 0} ; P\right\}$ with stable branching mechanism $\psi(z)=c z^{\gamma}$, where $c>0$ and $\gamma \in(1,2]$. They showed that for all $x \geq 0$, with $c_{t}:=(c(\gamma-1) t)^{1 /(\gamma-1)}$,

$$
\left\{c_{t}^{-1} Y_{t} ; P\left(\cdot \mid Y_{t}>0, Y_{0}=x\right)\right\} \underset{t \rightarrow \infty}{\stackrel{\text { law }}{\longrightarrow}} \mathbf{z}^{(\gamma-1)},
$$

where $\mathbf{z}^{(\gamma-1)}$ is a random variable with Laplace transform given by (1.6) (with $\alpha=\gamma-1$ ) and is independent of the initial position $x$. Recently, Ren, Yang and Zhao [28] studied CSBPs $\left\{\left(Y_{t}\right)_{t \geq 0} ; P\right\}$ with branching mechanism

$$
\psi(z)=c z^{\gamma} l(z), \quad z \geq 0,
$$

where $c>0, \gamma \in(1,2]$ and $l$ is a function slowly varying at 0 . It was proved in [28] that for all $x \geq 0$, with $\lambda_{t}:=P_{1}\left(Y_{t}>0\right)$,

$$
\left\{\lambda_{t} Y_{t} ; P\left(\cdot \mid Y_{t}>0, Y_{0}=x\right)\right\} \underset{t \rightarrow \infty}{\stackrel{\text { law }}{\longrightarrow}} \mathbf{z}^{(\gamma-1)}
$$

where the distribution of the random variable $\mathbf{z}^{(\gamma-1)}$ is given by (1.6) (with $\alpha=\gamma-1$ ) and is independent of the initial position $x$.

Later, Iyer, Leger and Pego [11] considered the converse problem: Suppose $\left\{\left(Y_{t}\right)_{t \geq 0} ; P\right\}$ is a CSBP with critical branching mechanism $\psi$ satisfying Grey's condition. In order for the left side of (1.16) to have a non-trivial weak limit for some positive constants $\left(\lambda_{t}\right)_{t \geq 0}$, one must have (1.15) for some $1<\gamma \leq 2$.

In this paper, we will establish a result parallel to (1.14) for some critical $(\xi, \psi)$ superprocesses $\{X ; \mathbf{P}\}$ with spatially dependent stable branching mechanism. In particular, we assume that the spatial motion $\xi$ is intrinsically ultracontractive with respect to some reference measure $m$, and the branching mechanism takes the form

$$
\begin{aligned}
\psi(x, z) & =-\beta(x) z+\kappa(x) \int_{0}^{\infty}\left(e^{-z y}-1+z y\right) \frac{d y}{\Gamma(-\gamma(x)) y^{1+\gamma(x)}} \\
& =-\beta(x) z+\kappa(x) z^{\gamma(x)}, \quad x \in E, z \geq 0,
\end{aligned}
$$

where $\beta \in \mathscr{B}_{b}(E), \gamma \in \mathscr{B}_{b}^{+}(E), \kappa \in \mathscr{B}_{b}^{+}(E)$ with $1<\gamma(\cdot)<2, \gamma_{0}:=\operatorname{essinf}_{m(d x)} \gamma(x)>1$ and ess inf $m_{m(d x)} \kappa(x)>0$. Let $\mu$ be an arbitrary finite initial measure on $E$. We will show that $\mathbf{P}_{\mu}\left(\left\|X_{t}\right\| \neq 0\right)$ converges to 0 as $t \rightarrow \infty$ and is regularly varying at infinity with index $\frac{1}{\gamma_{0}-1}$. Furthermore, if $m\left(x: \gamma(x)=\gamma_{0}\right)>0$, we will show that

$$
\lim _{t \rightarrow \infty} \eta_{t}^{-1} \mathbf{P}_{\mu}\left(\left\|X_{t}\right\| \neq 0\right)=\mu(\phi)
$$

and for a large class of non-negative testing functions $f$,

$$
\left\{\eta_{t} X_{t}(f) ; \mathbf{P}_{\mu}\left(\cdot \mid\left\|X_{t}\right\| \neq 0\right)\right\} \underset{t \rightarrow \infty}{\stackrel{\text { law }}{\longrightarrow}}\left\langle f, \phi^{*}\right\rangle_{m} \mathbf{z}^{\left(\gamma_{0}-1\right)},
$$


where $\eta_{t}:=\left(C_{X}\left(\gamma_{0}-1\right) t\right)^{-\frac{1}{\gamma_{0}-1}}, C_{X}:=\left\langle\mathbf{1}_{\gamma(\cdot)=\gamma_{0}} \kappa \cdot \phi^{\gamma_{0}}, \phi^{*}\right\rangle_{m}$ and $\mathbf{z}^{\left(\gamma_{0}-1\right)}$ is a random variable with Laplace transform given by (1.6) (with $\alpha=\gamma_{0}-1$ ). Notice that the distribution of the weak limit $\left\langle f, \phi^{*}\right\rangle_{m} \mathbf{z}^{\left(\gamma_{0}-1\right)}$ does not depend on $\mu$. Precise statements of the assumptions and the results are presented in the next subsection. It is interesting to mention here that, even though the stable index $\gamma(x)$ is spatially dependent, the limiting behavior of the critical superprocess $\{X ; \mathbf{P}\}$ depends primarily on the lowest index $\gamma_{0}$.

1.2. Model and results. We first fix our notation. Unless stated explicitly otherwise, $E$ is assumed to be a locally compact separable metric space. We use $\mathscr{B}(E)$ to denote the collection of all Borel subsets of $E$ and also the collection of all Borel functions on $E$. Define $\mathscr{B}_{b}(E):=\left\{f \in \mathscr{B}(E): \sup _{x \in E}|f(x)|<\infty\right\}, \mathscr{B}^{+}(E):=\{f \in \mathscr{B}(E): \forall x \in$ $E, f(x) \geq 0\}$ and $\mathscr{B}^{++}(E):=\{f \in \mathscr{B}(E): \forall x \in E, f(x)>0\}$. Define $\mathscr{B}_{b}^{+}(E):=$ $\mathscr{B}_{b}(E) \cap \mathscr{B}^{+}(E)$ and $\mathscr{B}_{b}^{++}(E):=\mathscr{B}_{b}(E) \cap \mathscr{B}^{++}(E)$. Denote by $\mathcal{M}_{E}$ the collection of all Borel measures on $E$. Denote by $\mathcal{M}_{E}^{\sigma}$ the collection of all $\sigma$-finite Borel measures on $E$. For simplicity, we write $\mu(f)$ and sometimes $\langle\mu, f\rangle$ for the integration of a function $f$ with respect to a measure $\mu$. For any $f \in \mathscr{B}^{+}(E)$, define $\mathcal{M}_{E}^{f}:=\left\{\mu \in \mathcal{M}_{E}: \mu(f)<\infty\right\}$. In particular, $\mathcal{M}_{E}^{1}$ is the collection of all finite Borel measures on $E$.

We now give the definition of a $(\xi, \psi)$-superprocess: Let the spatial motion $\xi=$ $\left\{\left(\xi_{t}\right)_{t \geq 0} ;\left(\Pi_{x}\right)_{x \in E}\right\}$ be an $E$-valued Hunt process with its lifetime denoted by $\zeta$, and the branching mechanism $\psi$ be a function on $E \times[0, \infty)$ given by (1.10). We say an $\mathcal{M}_{E}^{1}$-valued Hunt process $X=\left\{\left(X_{t}\right)_{t \geq 0} ;\left(\mathbf{P}_{\mu}\right)_{\mu \in \mathcal{M}_{E}^{1}}\right\}$ is a $(\xi, \psi)$-superprocess if for each $t \geq 0, \mu \in \mathcal{M}_{E}^{1}$ and $f \in \mathscr{B}_{b}^{+}(E)$, we have

$$
\mathbf{P}_{\mu}\left[e^{-X_{t}(f)}\right]=e^{-\mu\left(V_{t} f\right)},
$$

where the function $(t, x) \mapsto V_{t} f(x)$ on $[0, \infty) \times E$ is the unique locally bounded positive solution to the equation

$$
V_{t} f(x)+\Pi_{x}\left[\int_{0}^{t \wedge \zeta} \psi\left(\xi_{s}, V_{t-s} f\right) d s\right]=\Pi_{x}\left[f\left(\xi_{t}\right) \mathbf{1}_{t<\zeta}\right], \quad t \geq 0, x \in E .
$$

(In this paper, for any real-valued function $F$ on $E \times[0, \infty)$ and real-valued function $f$ on $E$, we write $F(x, f):=F(x, f(x))$ for simplicity.)

Recall that the branching mechanism $\psi$ is given by (1.10) and its linear coefficient $\beta$ is a bounded Borel function on E. Define the Feynman-Kac semigroup

$$
P_{t}^{\beta} f(x):=\Pi_{x}\left[e^{\int_{0}^{t} \beta\left(\xi_{r}\right) d r} f\left(\xi_{t}\right) \mathbf{1}_{t<\zeta}\right], \quad t \geq 0, x \in E, f \in \mathscr{B}_{b}(E) .
$$

It is known, see [20, Proposition 2.27] for example, $\left(P_{t}^{\beta}\right)$ is the mean semigroup of the superprocess $\{X ; \mathbf{P}\}$ in the sense that

$$
\mathbf{P}_{\mu}\left[X_{t}(f)\right]=\mu\left(P_{t}^{\beta} f\right), \quad \mu \in \mathcal{M}_{E}^{1}, t \geq 0, f \in \mathscr{B}_{b}(E) .
$$

The mean semigroup plays a central role in the study of the asymptotic behavior of superprocesses. As discussed in [8], in order to have a result like (1.13) or (1.17), we have to establish the asymptotic behavior of the mean semigroup first. This can be done under the following assumptions on the spatial motion $\xi$ : 
Assumption 1. There exist an $m \in \mathcal{M}_{E}^{\sigma}$ with full support on the state space $E$ and a family of strictly positive, bounded continuous functions $\left\{p_{t}(\cdot, \cdot): t>0\right\}$ on $E \times E$ such that

$$
\begin{gathered}
\Pi_{x}\left[f\left(\xi_{t}\right) \mathbf{1}_{t<\zeta}\right]=\int_{E} p_{t}(x, y) f(y) m(d y), \quad t>0, x \in E, f \in \mathscr{B}_{b}(E) ; \\
\int_{E} p_{t}(y, x) m(d y) \leq 1, \quad t>0, x \in E ; \\
\int_{E} \int_{E} p_{t}(x, y)^{2} m(d x) m(d y)<\infty, \quad t>0 ;
\end{gathered}
$$

and the functions $x \mapsto \int_{E} p_{t}(x, y)^{2} m(d y)$ and $x \mapsto \int_{E} p_{t}(y, x)^{2} m(d y)$ are both continuous.

We will write $\langle f, g\rangle_{m}$ for $\int_{E} f g d m$ to emphasize that it is the inner product in the Hilbert space $L^{2}(E, m)$. Let $\left(P_{t}^{*}\right)_{t \geq 0}$ be the dual of the transition semigroup $\left(P_{t}\right)_{t \geq 0}$ in $L^{2}(E, m)$, i.e.,

$$
P_{0}^{*}=I ; \quad P_{t}^{*} f(x):=\int_{E} p_{t}(y, x) f(y) m(d y), \quad t>0, x \in E, f \in \mathscr{B}_{b}(E) .
$$

Under Assumption 1, it is proved in [26] and [27] that $\left(P_{t}\right)_{t \geq 0}$ and $\left(P_{t}^{*}\right)_{t \geq 0}$ are both strongly continuous semigroups of compact operators on $L^{2}(E, m)$. Let $\widetilde{L}$ and $\widetilde{L}^{*}$ be the generators of the semigroups $\left(P_{t}\right)_{t \geq 0}$ and $\left(P_{t}^{*}\right)_{t \geq 0}$, respectively. Denote by $\sigma(\widetilde{L})$ and $\sigma\left(\widetilde{L}^{*}\right)$ the spectra of $\widetilde{L}$ and $\widetilde{L}^{*}$, respectively. According to [29, Theorem V.6.6], $\widetilde{\lambda}:=$ $\sup \operatorname{Re}(\sigma(\widetilde{L}))=\sup \operatorname{Re}\left(\sigma\left(\widetilde{L}^{*}\right)\right)$ is a common eigenvalue of multiplicity 1 for both $\widetilde{L}$ and $\widetilde{L}^{*}$. By the argument in [26], the eigenfunctions $\widetilde{\phi}$ of $\widetilde{L}$ and $\widetilde{\phi}^{*}$ of $\widetilde{L}^{*}$ associated with the eigenvalue $\widetilde{\lambda}$ can be chosen to be strictly positive and continuous everywhere on $E$. We further normalize $\widetilde{\phi}$ and $\widetilde{\phi}^{*}$ by $\langle\widetilde{\phi}, \widetilde{\phi}\rangle_{m}=\left\langle\widetilde{\phi}, \widetilde{\phi}^{*}\right\rangle_{m}=1$ so that they are unique.

It is also proved in [26, 27] that there exists a function $p_{t}^{\beta}(x, y)$ on $(0, \infty) \times E \times E$ which is continuous in $(x, y)$ for each $t>0$ such that

$$
e^{-\|\beta\|_{\infty} t} p_{t}(x, y) \leq p_{t}^{\beta}(x, y) \leq e^{\|\beta\|_{\infty} t} p_{t}(x, y), \quad t>0, x, y \in E,
$$

and that for any $t>0, x \in E$ and $f \in \mathscr{B}_{b}(E)$,

$$
P_{t}^{\beta} f(x)=\int_{E} p_{t}^{\beta}(x, y) f(y) m(d y) .
$$

$\left(p_{t}^{\beta}\right)_{t \geq 0}$ is called the density of the semigroup $\left(P_{t}^{\beta}\right)_{t \geq 0}$. Define the dual semigroup $\left(P_{t}^{\beta *}\right)_{t \geq 0}$ by

$$
P_{0}^{\beta *}=I ; \quad P_{t}^{\beta *} f(x):=\int_{E} p_{t}^{\beta}(y, x) f(y) m(d y), \quad t>0, x \in E, f \in \mathscr{B}_{b}(E) .
$$

It is proved in [26, 27] that $\left(P_{t}^{\beta}\right)_{t \geq 0}$ and $\left(P_{t}^{\beta *}\right)_{t \geq 0}$ are both strongly continuous semigroups of compact operators on $L^{2}(E, m)$. Let $L$ and $L^{*}$ be the generators of the semigroups $\left(P_{t}^{\beta}\right)_{t \geq 0}$ and $\left(P_{t}^{\beta *}\right)_{t \geq 0}$, respectively. Denote by $\sigma(L)$ and $\sigma\left(L^{*}\right)$ the spectra of $L$ and $L^{*}$, respectively. According to [29, Theorem V.6.6], $\lambda:=\sup \operatorname{Re}(\sigma(L))=\sup \operatorname{Re}\left(\sigma\left(L^{*}\right)\right)$ is a common eigenvalue of multiplicity 1 for both $L$ and $L^{*}$. By the argument in [26], the 
eigenfunctions $\phi$ of $L$ and $\phi^{*}$ of $L^{*}$ associated with the eigenvalue $\lambda$ can be chosen to be strictly positive and continuous everywhere on $E$. We further normalize $\phi$ and $\phi^{*}$ by $\langle\phi, \phi\rangle_{m}=\left\langle\phi, \phi^{*}\right\rangle_{m}=1$ so that they are unique. Moreover, for each $t \geq 0$ and $x \in E$, we have $P_{t}^{\beta} \phi(x)=e^{\lambda t} \phi(x)$ and $P_{t}^{\beta *} \phi^{*}(x)=e^{\lambda t} \phi^{*}(x)$. We refer to $\phi\left(\right.$ resp. $\left.\phi^{*}\right)$ and $\lambda$ as the principal eigenfunction and the principal eigenvalue of $L$ (resp. $\left.L^{*}\right)$.

Now, from

$$
\mathbf{P}_{\mu}\left[X_{t}(\phi)\right]=e^{\lambda t} \mu(\phi), \quad t \geq 0,
$$

we see that, if $\lambda>0$, the mean of $X_{t}(\phi)$ will increase exponentially; if $\lambda<0$, the mean of $X_{t}(\phi)$ will decrease exponentially; and if $\lambda=0$, the mean of $X_{t}(\phi)$ will be a constant. Therefore, we say $X$ is supercritical, critical or subcritical, according to $\lambda>0, \lambda=0$ or $\lambda<0$, respectively. Since we are only interested in the critical case, we assume the following:

Assumption 2. The superprocess $X$ is critical, i.e., $\lambda=0$.

Our next assumption is on the spatial motion $\xi$ :

Assumption 3. $\widetilde{\phi}$ is bounded, and $\left(P_{t}\right)_{t \geq 0}$ is intrinsically ultracontractive, that is, for each $t>0$, there is a constant $c_{t}>0$ such that for each $x, y \in E, p_{t}(x, y) \leq c_{t} \widetilde{\phi}(x) \widetilde{\phi}^{*}(y)$.

Under Assumption 3, it is proved in [26, 27] that the principal eigenfunction $\phi$ of the Feynman-Kac semigroup $\left(P_{t}^{\beta}\right)_{t \geq 0}$ is also bounded. Moreover, $\left(P_{t}^{\beta}\right)_{t \geq 0}$ is also intrinsically ultracontractive, in the sense that for each $t>0$, there is a constant $c_{t}>0$ such that for each $x, y \in E, p_{t}^{\beta}(x, y) \leq c_{t} \phi(x) \phi^{*}(y)$. In fact, it is proved in [14 that for each $t>0$, $\left(p_{t}^{\beta}(x, y)\right)_{x, y \in E}$ is comparable to $\left(\phi(x) \phi^{*}(y)\right)_{x, y \in E}$ in the sense that there is a constant $c_{t}>1$ such that

$$
c_{t}^{-1} \leq \frac{p_{t}^{\beta}(x, y)}{\phi(x) \phi^{*}(y)} \leq c_{t}, \quad x, y \in E .
$$

It is also shown in [14 that there are constants $c_{0}, c_{1}>0$ such that

$$
\sup _{x, y \in E}\left|\frac{p_{t}^{\beta}(x, y)}{\phi(x) \phi^{*}(y)}-1\right| \leq c_{0} e^{-c_{1} t}, \quad t>1 .
$$

Assumption 3 is a pretty strong assumption on the semigroup $\left\{P_{t}: t \geq 0\right\}$. For example, it rules out the semigroup of Brownian motion on $\mathbb{R}^{d}$ and the semgroup of OrnsteinUhlenbeck process on $\mathbb{R}^{d}$. However, this assumption is satisfied in a lot of cases. In [26], a list of examples of processes satisfying Assumptions 1 and 3 were given. For the convenience of our readers, we will briefly recall some of these examples in Subsection A.1.

Recall that the branching mechanism is given by (1.10). We assume the following:

Assumption 4. The branching mechanism $\psi$ is of the form:

$$
\psi(x, z)=-\beta(x) z+\kappa(x) \int_{0}^{\infty}\left(e^{-z y}-1+z y\right) \frac{d y}{\Gamma(-\gamma(x)) y^{1+\gamma(x)}}
$$




$$
=-\beta(x) z+\kappa(x) z^{\gamma(x)}, \quad x \in E, z \geq 0,
$$

where $\gamma \in \mathscr{B}_{b}^{+}(E), \kappa \in \mathscr{B}_{b}^{++}(E)$ with $1<\gamma(\cdot)<2, \gamma_{0}:=\operatorname{essinf}_{m(d x)} \gamma(x)>1$ and $\kappa_{0}:=\operatorname{ess~inf}_{m(d x)} \kappa(x)>0$.

Here we used the definition of the Gamma function on the negative half line:

$$
\Gamma(x):=\int_{0}^{\infty} t^{x-1}\left(e^{-t}-\sum_{k=0}^{n-1} \frac{(-t)^{k}}{k !}\right) d t, \quad-n<x<-n+1, n \in \mathbb{N} .
$$

We now present the main result of this paper:

Theorem 1.1. Suppose that $\left\{\left(X_{t}\right)_{t \geq 0} ;\left(\mathbf{P}_{\mu}\right)_{\mu \in \mathcal{M}_{E}^{1}}\right\}$ is a $(\xi, \psi)$-superprocess satisfying Assumptions 14. Then,

(1) $\{X ; \mathbf{P}\}$ is non-persistent, that is, for each $t>0$ and $x \in E, \mathbf{P}_{\delta_{x}}\left(\left\|X_{t}\right\|=0\right)>0$.

(2) For each $\mu \in \mathcal{M}_{E}^{1}, \mathbf{P}_{\mu}\left(\left\|X_{t}\right\| \neq 0\right)$ converges to 0 as $t \rightarrow \infty$ and is regularly varying at infinity with index $-\left(\gamma_{0}-1\right)^{-1}$. Furthermore, if $m\left(x: \gamma(x)=\gamma_{0}\right)>0$, then

$$
\lim _{t \rightarrow \infty} \eta_{t}^{-1} \mathbf{P}_{\mu}\left(\left\|X_{t}\right\| \neq 0\right)=\mu(\phi) .
$$

(3) Suppose $m\left(x: \gamma(x)=\gamma_{0}\right)>0$. Let $f \in \mathscr{B}^{+}(E)$ be such that $\left\langle f, \phi^{*}\right\rangle_{m}>0$ and $\left\|\phi^{-1} f\right\|_{\infty}<\infty$. Then for each $\mu \in \mathcal{M}_{E}^{1}$,

$$
\left\{\eta_{t} X_{t}(f) ; \mathbf{P}_{\mu}\left(\cdot \mid\left\|X_{t}\right\| \neq 0\right)\right\} \underset{t \rightarrow \infty}{\stackrel{\text { law }}{\longrightarrow}}\left\langle f, \phi^{*}\right\rangle_{m} \mathbf{z}^{\left(\gamma_{0}-1\right)} \text {. }
$$

Here, $\eta_{t}:=\left(C_{X}\left(\gamma_{0}-1\right) t\right)^{-\frac{1}{\gamma_{0}-1}}, C_{X}:=\left\langle\mathbf{1}_{\gamma(\cdot)=\gamma_{0}} \kappa \cdot \phi^{\gamma_{0}}, \phi^{*}\right\rangle_{m}$ and $\mathbf{z}^{\left(\gamma_{0}-1\right)}$ is a random variable with Laplace transform given by (1.6) (with $\left.\alpha=\gamma_{0}-1\right)$.

1.3. Methods and overview. To establish Theorem 1.1(2) and Theorem 1.1](3), we use a spine decomposition theorem for $X$. Roughly speaking, the spine is the trajectory of an immortal moving particle and the spine decomposition theorem says that, after a martingale change of measure, the transformed superprocess can be decomposed in law as the sum of a copy of the original superprocess and a measure-valued immigration process along this spine, see [6, 7, 21]. The martingale used for the change of measure is $\left(e^{-\lambda t} X_{t}(\phi)\right)_{t \geq 0}$. Under Assumptions 1 and 3 , the spine process $\left\{\xi ; \Pi^{(\phi)}\right\}$ is an ergodic process. We take advantage of this ergodicity to study the asymptotic behavior of the superprocess.

Similar idea has already been used by Powell [23] to establish results parallel to (1.12) and (1.13) for a class of critical branching diffusion processes. Let $\left\{\left(Y_{t}\right)_{t \geq 0} ; P\right\}$ be a branching diffusion process in a bounded domain with finite second moment. As have been discussed in [23], a direct study of the partial differential equation satisfied by the survival probability $(t, x) \mapsto P_{\delta_{x}}\left(\left\|Y_{t}\right\| \neq 0\right)$ is tricky. Instead, by using a spine decomposition approach, Powell [23] showed that the survival probability decays like $a(t) \phi(x)$, where $\phi(x)$ is the principal eigenfunction of the mean semigroup of $\left(Y_{t}\right)$ and $a(t)$ is a function capturing the uniform speed. Then the problem is reduced to the study of a single ordinary differential equation satisfied by $a(t)$. Later, inspired by [23], 
we gave in [25] a similar proof of (1.12) for a class of general critical superprocesses with finite second moment. In this paper, we will generalize these arguments to a class of general critical superprocesses without finite second moment and establish Theorem 1.1(2). For the conditional weak convergence result, i.e., Theorem 1.1(3), we use a fact that the Laplace transform given in (1.6) can be characterized by a non-linear delay equation (see Lemma A.9). Using the spine method, we show that the Laplace transform of the one-dimensional distributions of the superprocess, after a proper rescaling, can be characterized by a similar equation (see (3.24)). Then, the desired convergence of the distributions can be established by a comparison between the equations. Again, the ergodicity of the spine process plays a central role in the comparison.

A similar idea for establishing weak convergence through a comparison of the equations satisfied by the distributions has already been used by us in [24, 25]. We characterized the exponential distribution using its double size-biased transform; and to help us make the comparison, we investigated the double size-biased transform of the corresponding processes. However, the double-size-biased transform of a random variable requires its second moment being finite. Since we do not assume the second moment condition in this paper, we can not use the method of double size-biased transform.

In [23] (for critical branching diffusions in a bounded domain with finite variance) and in [25, 26] (for general critical superprocesses with finite variance), the conditional weak convergence was proved in two steps. First, a convergence result was established for $\phi$, the principal eigenfunction of the mean semigroup of the corresponding process, and then the second moment condition was used to extend the result to more general testing functions. However, in the present case, since we are not assuming the second moment condition, this type of argument does not work. Instead, we use a generalized spine decomposition theorem, which is developed in [25], to establish Theorem 1.1)(3) for a large class of general testing functions in one stroke.

The rest of this paper is organized as follows: In Subsections 2.1, 2.2 and 2.3, we give some preliminary results about the asymptotic equivalence, regularly variation and superprocesses, respectively. In Subsection 2.4, we present the generalized spine decomposition theorem. In Subsection 2.5, we discuss the ergodicity of the spine process. In Subsections 3.1 and 3.2 we give the poofs of Theorem 1.1(1) and 1.1(2), respectively. In Subsection 3.3, we give the equation that characterize the one-dimensional distributions. In Subsection 3.4, we give the proof of Theorem 1.1(3). In Appendix A.2, we give the equation that characterizes the distribution with Laplace transform (1.6), which is used in the proof of Theorem 1.1(3).

\section{Preliminaries}

2.1. Asymptotic equivalence. In this subsection, we give a lemma on asymptotic equivalence. Let $t_{0} \in[-\infty, \infty]$. In this subsection, $(E, \mathscr{E})$ is assumed to be a measurable space. For any $f_{0}, f_{1} \in \mathscr{B}^{++}(\mathbb{R})$, we say $f_{0}$ and $f_{1}$ are asymptotically equivalent 
at $t_{0}$, if $\left|\frac{f_{0}(t)}{f_{1}(t)}-1\right| \underset{t \rightarrow t_{0}}{\longrightarrow} 0$; and in this case, we write $f_{0}(t) \underset{t \rightarrow t_{0}}{\sim} f_{1}(t)$. For any strictly positive measurable functions $g_{0}, g_{1}$ on $\mathbb{R} \times E$, we say $g_{0}$ and $g_{1}$ are uniformly asymptotically equivalent at $t_{0}$, if $\sup _{x \in E}\left|\frac{g_{0}(t, x)}{g_{1}(t, x)}-1\right| \underset{t \rightarrow t_{0}}{\longrightarrow} 0$; and in this case, we write $g_{0}(t, x) \underset{t \rightarrow t_{0}}{\stackrel{x \in E}{\sim}} g_{1}(t, x)$.

Lemma 2.1. Suppose that $f_{0}, f_{1}$ are bounded strictly positive measurable functions on $\mathbb{R} \times E$ and $f_{0}(t, x) \underset{t \rightarrow t_{0}}{\stackrel{x \in E}{\in}} f_{1}(t, x)$. If $\rho$ is a finite non-degenerate measure on $(E, \mathscr{E})$, then

$$
\int_{E} f_{0}(t, x) \rho(d x) \underset{t \rightarrow t_{0}}{\sim} \int_{E} f_{1}(t, x) \rho(d x) .
$$

Proof. Since

$$
\begin{aligned}
& \left|\frac{\int_{E} f_{0}(t, x) \rho(d x)}{\int_{E} f_{1}(t, x) \rho(d x)}-1\right|=\left|\int_{E} \frac{f_{0}(t, x)}{f_{1}(t, x)} \frac{f_{1}(t, x) \rho(d x)}{\int_{E} f_{1}(t, y) \rho(d y)}-1\right| \\
& \quad \leq \int_{E}\left|\frac{f_{0}(t, x)}{f_{1}(t, x)}-1\right| \frac{f_{1}(t, x) \rho(d x)}{\int_{E} f_{1}(t, y) \rho(d y)} \leq \sup _{x \in E}\left|\frac{f_{0}(t, x)}{f_{1}(t, x)}-1\right| \underset{t \rightarrow t_{0}}{\longrightarrow} 0
\end{aligned}
$$

the assertion is valid.

2.2. Regular variation. In this subsection, we give some preliminary results on regular variation. We refer the reader to [4] for more results on regular variation. For $f \in$ $\mathscr{B}^{++}((0, \infty))$ and $\alpha \in(-\infty, \infty)$, we say $f$ is regularly varying at $\infty$ (resp. at 0 ) with index $\alpha$ if for any $u \in(0, \infty)$,

$$
\lim _{t \rightarrow \infty} \frac{f(u t)}{f(t)}=u^{\alpha} \quad\left(\text { resp. } \lim _{t \rightarrow 0} \frac{f(u t)}{f(t)}=u^{\alpha}\right) .
$$

In this case we write $f \in \mathcal{R}_{\alpha}^{\infty}$ (resp. $f \in \mathcal{R}_{\alpha}^{0}$ ). Further, if $\alpha=0$, then we say $f$ is slowly varying. According to [4, Theorem 1.3.1], if $L$ is a function slowly varying at $\infty$, then it can be written in the form

$$
L(t)=c(t) \exp \left\{\int_{t_{0}}^{t} \epsilon(u) \frac{d u}{u}\right\}, \quad t \geq t_{0},
$$

for some $t_{0}>0$, where $(c(t))_{t \geq t_{0}}$ and $(\epsilon(t))_{t \geq t_{0}}$ are measurable functions with $c(t) \underset{t \rightarrow \infty}{\longrightarrow}$ $c \in(0, \infty)$ and $\epsilon(t) \underset{t \rightarrow \infty}{\longrightarrow} 0$. In particular, we know that, there is $t_{0}>0$ large enough such that $L$ is locally bounded on $\left[t_{0}, \infty\right)$.

Lemma 2.2 ([4, Propositions 1.5.8 and 1.5.10]). Suppose that $L \in \mathcal{R}_{0}^{\infty}$.

- Let $t_{0} \in(0, \infty)$ be large enough so that $L$ is locally bounded on $\left[t_{0}, \infty\right)$. If $\alpha>0$, then

$$
\int_{t_{0}}^{t} L(u) d u^{\alpha} \underset{t \rightarrow \infty}{\sim} t^{\alpha} L(t) .
$$

- If $\alpha<0$ then $\int_{t}^{\infty} L(u) d u^{\alpha}<\infty$ for $t$ large enough, and

$$
-\int_{t}^{\infty} L(u) d u^{\alpha} \underset{t \rightarrow \infty}{\sim} t^{\alpha} L(t)
$$


Corollary 2.3. Suppose that $l \in \mathcal{R}_{0}^{0}$.

- Let $s_{0} \in(0, \infty)$ be small enough so that $l$ is locally bounded on $\left(0, s_{0}\right]$. If $\alpha<0$, then

$$
-\int_{s}^{s_{0}} l(u) d u^{\alpha} \underset{s \rightarrow 0}{\sim} s^{\alpha} l(s) .
$$

- If $\alpha>0$, then $\int_{0}^{s} l(u) d u^{\alpha}<\infty$ for s small enough, and

$$
\int_{0}^{s} l(u) d u^{\alpha} \underset{s \rightarrow 0}{\sim} s^{\alpha} l(s) .
$$

Proof. Since $l \in \mathcal{R}_{0}^{0}$, we know that, if one defines $L(t):=l\left(t^{-1}\right)$ for each $t \in(0, \infty)$, then $L \in \mathcal{R}_{0}^{\infty}$. Therefore, there exists $t_{0} \in(0, \infty)$ such that $L$ is locally bounded on $\left[t_{0}, \infty\right)$. Taking $s_{0}:=t_{0}^{-1}$, we then immediately get that $l$ is locally bounded on $\left(0, s_{0}\right]$. If $\alpha<0$, then according to Lemma 2.2 , we have

$$
\int_{t_{0}}^{t} L(u) d u^{-\alpha} \underset{t \rightarrow \infty}{\sim} t^{-\alpha} L(t) .
$$

Replacing $t$ with $s^{-1}$, we have

$$
-\int_{s}^{s_{0}} l(u) d u^{\alpha}=\int_{s_{0}^{-1}}^{s^{-1}} L(u) d u^{-\alpha} \underset{s \rightarrow 0}{\sim}\left(s^{-1}\right)^{-\alpha} L\left(s^{-1}\right)=s^{\alpha} l(s),
$$

as desired. The second assertion can be proved similarly.

Lemma 2.4 ([4, Theorem 1.5.12]). If $f \in \mathcal{R}_{\alpha}^{\infty}$ with $\alpha>0$, there exists $g \in \mathcal{R}_{1 / \alpha}^{\infty}$ with

$$
g(f(t)) \underset{t \rightarrow \infty}{\sim} f(g(t)) \underset{t \rightarrow \infty}{\sim} t .
$$

Here $g$ is determined uniquely up to asymptotic equivalence as $t \rightarrow \infty$.

Corollary 2.5. If $f \in \mathcal{R}_{\alpha}^{0}$ with $\alpha<0$, there exists $g \in \mathcal{R}_{1 / \alpha}^{\infty}$ with

$$
g(f(t)) \underset{t \rightarrow 0}{\sim} t ; \quad f(g(t)) \underset{t \rightarrow \infty}{\sim} t .
$$

Here $g$ is determined uniquely up to asymptotic equivalence as $t \rightarrow \infty$.

Proof. Since $f \in \mathcal{R}_{\alpha}^{0}$, we know that $\tilde{f} \in \mathcal{R}_{-\alpha}^{\infty}$ with $\widetilde{f}(t):=f\left(t^{-1}\right)$. Noticing that $-\alpha>0$, according to Lemma 2.4, there exists $h \in \mathcal{R}_{-1 / \alpha}^{\infty}$ such that

$$
h(\widetilde{f}(t)) \underset{t \rightarrow \infty}{\sim} t ; \quad \widetilde{f}(h(t)) \underset{t \rightarrow \infty}{\sim} t .
$$

Denoting by $g:=h^{-1} \in \mathcal{R}_{1 / \alpha}^{\infty}$, the above translates to (2.1).

Now, suppose that there is another $g_{0} \in \mathcal{R}_{1 / \alpha}^{\infty}$ satisfies (2.1) with $g$ replaced by $g_{0}$. Denoting by $h_{0}:=g_{0}^{-1}$, we can verify that (2.2) is valid with $h$ replaced by $h_{0}$. According to Lemma 2.4, $h$ and $h_{0}$ are asymptotically equivalent at $\infty$. Hence, so are $g$ and $g_{0}$. 
Lemma 2.6. Let $(E, \mathscr{E})$ be a measurable space and $\rho$ a finite non-degenerate measure on $(E, \mathscr{E})$. Let $\alpha$ be a bounded measurable function on $E$ with

$$
\alpha_{0}:=\underset{\rho(d x)}{\operatorname{essinf}} \alpha(x):=\sup \{r: \rho(x: \alpha(x)<r)=0\} \in \mathbb{R} .
$$

Then $\left(\int_{E} t^{\alpha(x)} \rho(d x)\right)_{t \in(0, \infty)} \in \mathcal{R}_{\alpha_{0}}^{0}$. Further, if $\rho\left\{x: \alpha(x)=\alpha_{0}\right\}>0$, then

$$
\int_{E} t^{\alpha(x)} \rho(d x) \underset{t \rightarrow 0}{\sim} \rho\left\{x: \alpha(x)=\alpha_{0}\right\} t^{\alpha_{0}} .
$$

Proof. If $u \in(0,1]$, then we have

$$
\frac{\int_{E} u^{\alpha(x)} t^{\alpha(x)} \rho(d x)}{\int_{E} t^{\alpha(x)} \rho(d x)} \leq \frac{\int_{E} u^{\alpha_{0}} t^{\alpha(x)} \rho(d x)}{\int_{E} t^{\alpha(x)} \rho(d x)}=u^{\alpha_{0}}, \quad t \in(0, \infty) .
$$

This implies that

$$
\limsup _{(0, \infty) \ni t \rightarrow 0} \frac{\int_{E} u^{\alpha(x)} t^{\alpha(x)} \rho(d x)}{\int_{E} t^{\alpha(x)} \rho(d x)} \leq u^{\alpha_{0}} .
$$

Also, for any $\epsilon \in(0, \infty)$, we have

$$
\begin{aligned}
& \frac{\int_{E} u^{\alpha(x)} t^{\alpha(x)} \rho(d x)}{\int_{E} t^{\alpha(x)} \rho(d x)} \geq \frac{\int_{\alpha(x) \leq \alpha_{0}+\epsilon} u^{\alpha(x)} t^{\alpha(x)} \rho(d x)}{\int_{E} t^{\alpha(x)} \rho(d x)} \\
& \quad \geq u^{\alpha_{0}+\epsilon} \frac{\int_{\alpha(x) \leq \alpha_{0}+\epsilon} t^{\alpha(x)} \rho(d x)}{\int_{\alpha(x) \leq \alpha_{0}+\epsilon} t^{\alpha(x)} \rho(d x)+\int_{\alpha(x)>\alpha_{0}+\epsilon} t^{\alpha(x)} \rho(d x)} \\
& \quad=u^{\alpha_{0}+\epsilon} \frac{1}{1+\frac{\int_{\alpha(x)>\alpha_{0}+\epsilon} t^{\alpha(x)-\left(\alpha_{0}+\epsilon\right)} \rho(d x)}{\int_{\alpha(x) \leq \alpha_{0}+\epsilon} t^{\alpha(x)-\left(\alpha_{0}+\epsilon\right)} \rho(d x)}}, \quad t \in(0, \infty), \\
& \underset{(0, \infty) \ni t \rightarrow 0}{\longrightarrow} u^{\alpha_{0}+\epsilon},
\end{aligned}
$$

where the last convergence is due to the monotone convergence theorem. Therefore

$$
\liminf _{(0, \infty) \ni t \rightarrow 0} \frac{\int_{E} u^{\alpha(x)} t^{\alpha(x)} \rho(d x)}{\int_{E} t^{\alpha(x)} \rho(d x)} \geq u^{\alpha_{0}} .
$$

Summarizing the above, we get

$$
\lim _{(0, \infty) \ni t \rightarrow 0} \frac{\int_{E} u^{\alpha(x)} t^{\alpha(x)} \rho(d x)}{\int_{E} t^{\alpha(x)} \rho(d x)}=u^{\alpha_{0}}, \quad u \in(0,1] .
$$

If $u \in(1, \infty)$, taking $f(x, t):=t^{\alpha(x)}$, from what we have proved, we also have that

$$
\lim _{(0, \infty) \ni t \rightarrow 0} \frac{\int_{E} f(x, u t) \rho(d x)}{\int_{E} f(x, t) \rho(d x)}=\lim _{(0, \infty) \ni t \rightarrow 0} \frac{\int_{E} f(x, t) \rho(d x)}{\int_{E} f\left(x, u^{-1} t\right) \rho(d x)}=\left(\left(u^{-1}\right)^{\alpha_{0}}\right)^{-1}=u^{\alpha_{0}} .
$$

This proved the first part of the lemma. 
If further we have $\rho\left(x: \alpha(x)=\alpha_{0}\right)>0$, then by the monotone convergence theorem it is easy to see that

$$
\frac{\int_{E} t^{\alpha(x)} \rho(d x)}{t^{\alpha_{0}}} \underset{(0, \infty) \ni t \rightarrow 0}{\longrightarrow} \rho\left(x: \alpha(x)=\alpha_{0}\right) \in(0, \infty) .
$$

2.3. Superprocesses. In this subsection, we recall some known results on the $(\xi, \psi)$ superprocess $\{X ; \mathbf{P}\}$. It is known, see [20, Theorem 2.23] for example, that (1.18) can be written as

$$
V_{t} f(x)+\int_{0}^{t} P_{t-r}^{\beta} \psi_{0}\left(x, V_{r} f\right) d r=P_{t}^{\beta} f(x), \quad f \in \mathscr{B}_{b}^{+}(E), t \geq 0, x \in E,
$$

where

$$
\psi_{0}(x, z):=\psi(x, z)+\beta(x) z, \quad x \in E, z \geq 0 .
$$

Suppose that Assumptions 12 hold. Since $\phi^{*}$ is the principal eigenfunction of the semigroup $\left(P_{t}^{\beta *}\right)_{t \geq 0}$, we have

$$
\left\langle P_{t}^{\beta} f, \phi^{*}\right\rangle_{m}=\left\langle f, P_{t}^{\beta *} \phi^{*}\right\rangle_{m}=\left\langle f, \phi^{*}\right\rangle_{m}, \quad f \in \mathscr{B}_{b}^{+}(E), t \geq 0 .
$$

Therefore, integrating both sides of (2.3) with respect to the measure $\phi^{*} d m$, we get that

$$
\left\langle V_{t} f, \phi^{*}\right\rangle_{m}+\int_{0}^{t}\left\langle\psi_{0}\left(\cdot, V_{r} f\right), \phi^{*}\right\rangle_{m} d r=\left\langle f, \phi^{*}\right\rangle_{m}, \quad t \geq 0, f \in \mathscr{B}_{b}^{+}(E) .
$$

This can be rearranged as

$$
\left\langle V_{t} f, \phi^{*}\right\rangle_{m}+\int_{s}^{t}\left\langle\psi_{0}\left(\cdot, V_{r} f\right), \phi^{*}\right\rangle_{m} d r=\left\langle V_{s} f, \phi^{*}\right\rangle_{m}, \quad t \geq s \geq 0, f \in \mathscr{B}_{b}^{+}(E) .
$$

Let $\mathbb{W}$ be the collection of all $\mathcal{M}_{E}^{1}$-valued càdlàg paths on $[0, \infty)$. We refer to $\mathbb{W}$ as the canonical space of $\left(X_{t}\right)_{t \geq 0}$. In fact, $\left(X_{t}\right)$ can be viewed as a $\mathbb{W}$-valued random variable. We denote the coordinate process of $\mathbb{W}$ by $\left(W_{t}\right)_{t \geq 0}$.

We say that $\left(X_{t}\right)_{t \geq 0}$ is non-persistent if $\mathbf{P}_{\delta_{x}}\left(\left\|X_{t}\right\|=0\right)>0$ for all $x \in E$ and $t>0$. Suppose that $\left(X_{t}\right)_{t \geq 0}$ is non-persistent, then according to [20, Section 8.4], there is a family of measures $\left(\mathbb{N}_{x}\right)_{x \in E}$ on $\mathbb{W}$ such that

- for each $x \in E, \mathbb{N}_{x}\left(\forall t>0,\left\|W_{t}\right\|=0\right)=0$;

- for each $x \in E, \mathbb{N}_{x}\left(\left\|W_{0}\right\| \neq 0\right)=0$;

- for any $\mu \in \mathcal{M}_{E}^{1}$, if $\mathcal{N}$ is a Poisson random measure on $\mathbb{W}$ with intensity $\mathbb{N}_{\mu}(\cdot):=$ $\int_{E} \mathbb{N}_{x}(\cdot) \mu(d x)$, then the superprocess $\left\{X ; \mathbf{P}_{\mu}\right\}$ can be realized by $\widetilde{X}_{0}:=\mu$ and $\widetilde{X}_{t}(\cdot):=\mathcal{N}\left[W_{t}(\cdot)\right], t>0$.

We refer to $\left(\mathbb{N}_{x}\right)_{x \in E}$ as the Kuznetsov measures of $X$. For the existence and further properties of such measures, we refer our readers to [20].

From Campbell's formula, see the proof of [18, Theorem 2.7] for example, we have

$$
-\log \mathbf{P}_{\mu}\left[e^{-X_{t}(f)}\right]=\mathbb{N}_{\mu}\left[1-e^{-W_{t}(f)}\right], \quad \mu \in \mathcal{M}_{E}^{1}, t>0, f \in \mathscr{B}_{b}^{+}(E) .
$$


For each $x \in E$ and $t \geq 0$, taking $\mu=\delta_{x}$ and $f=\lambda \mathbf{1}_{E}$ with $\lambda>0$ in the above equation, and letting $\lambda \rightarrow \infty$, we get

$$
v_{t}(x):=\lim _{\lambda \rightarrow \infty} V_{t}\left(\lambda \mathbf{1}_{E}\right)(x)=-\log \mathbf{P}_{\delta_{x}}\left(\left\|X_{t}\right\|=0\right)=\mathbb{N}_{x}\left(\left\|W_{t}\right\| \neq 0\right) .
$$

For each $\mu \in \mathcal{M}_{E}^{1}$ and $t>0$, by (2.5), (2.6) and the monotone convergence theorem, we have

$$
\begin{aligned}
\mathbb{N}_{\mu}\left(\left\|W_{t}\right\| \neq 0\right) & =-\log \mathbf{P}_{\mu}\left(\left\|X_{t}\right\|=0\right)=\lim _{\lambda \rightarrow \infty}\left(-\log \mathbf{P}_{\mu}\left[e^{-\lambda X_{t}\left(\mathbf{1}_{E}\right)}\right]\right) \\
& =\lim _{\lambda \rightarrow \infty}\left\langle\mu, V_{t}\left(\lambda \mathbf{1}_{E}\right)\right\rangle=\mu\left(v_{t}\right) .
\end{aligned}
$$

It is also known that for any $f \in \mathscr{B}_{b}^{+}(E)$,

$$
\mathbb{N}_{\mu}\left[W_{t}(f)\right]=\mathbf{P}_{\mu}\left[X_{t}(f)\right]=\mu\left(P_{t}^{\beta} f\right), \quad t \geq 0 .
$$

2.4. Spine decompositions. Let $(\Omega, \mathscr{F})$ be a measurable space with a $\sigma$-finite measure $\mu$. For any $F \in \mathscr{F}$, we say $\mu$ can be size-biased by $F$ if $\mu(F<0)=0$ and $\mu(F) \in(0, \infty)$. In this case, we define the $F$-transform of $\mu$ as the probability $\mu^{F}$ on $(\Omega, \mathscr{F})$ such that

$$
d \mu^{F}=\frac{F}{\mu(F)} d \mu .
$$

Let $\{X ; \mathbf{P}\}$ be a non-persistent superprocess. Let $\mu \in \mathcal{M}_{E}^{1}$ and $T>0$. Suppose that $g \in \mathscr{B}^{+}(E)$ satisfies that $\mu\left(P_{T}^{\beta} g\right) \in(0, \infty)$. Then, according to (2.8), $\mathbf{P}_{\mu}$ (resp. $\mathbb{N}_{\mu}$ ) can be size-biased by $X_{T}(g)$ (resp. $W_{T}(g)$ ). Denote by $\mathbf{P}_{\mu}^{X_{T}(g)}$ (resp. $\mathbb{N}_{\mu}^{W_{T}(g)}$ ) the $X_{T}(g)$ transform of $\mathbf{P}_{\mu}$ (resp. the $W_{T}(g)$-transform of $\mathbb{N}_{\mu}$ ). The spine decomposition theorem characterizes the law of $\left\{\left(X_{t}\right)_{t \geq 0} ; \mathbf{P}_{\mu}^{X_{T}(g)}\right\}$ in two steps. The first step of the theorem says that $\left\{\left(X_{t}\right)_{t \geq 0} ; \mathbf{P}_{\mu}^{X_{T}(g)}\right\}$ can be decomposed in law as the sum of two independent measure-valued processes:

Theorem 2.7 (Size-biased decomposition, [25]).

$$
\left\{\left(X_{t}\right)_{t \geq 0} ; \mathbf{P}_{\mu}^{X_{T}(g)}\right\} \stackrel{\text { f.d.d. }}{=}\left\{\left(X_{t}+W_{t}\right)_{t \geq 0} ; \mathbf{P}_{\mu} \otimes \mathbb{N}_{\mu}^{W_{T}(g)}\right\} .
$$

The second step of the spine decomposition theorem says that $\left\{\left(W_{t}\right)_{0 \leq t \leq T} ; \mathbb{N}_{\mu}^{W_{T}(g)}\right\}$ has a spine representation, which intuitively says that, under probability $\mathbb{N}_{\mu}^{W_{T}(g)}$, the measure-valued process $\left(W_{t}\right)_{0 \leq t \leq T}$ can be decomposed as a measure-valued immigration process along the trajectory of a spine process in a Poissonian way.

More precisely, we say $\left\{\left(\xi_{t}\right)_{0 \leq t \leq T}, \mathbf{n}_{T},\left(Y_{t}\right)_{0 \leq t \leq T} ; \dot{\mathbf{P}}_{\mu}^{(g, T)}\right\}$ is a spine representation of $\mathbb{N}_{\mu}^{W_{T}(g)}$ if:

- The spine process $\left\{\left(\xi_{t}\right)_{0 \leq t \leq T} ; \dot{\mathbf{P}}_{\mu}^{(g, T)}\right\}$ is a copy of $\left\{\left(\xi_{t}\right)_{0 \leq t \leq T} ; \Pi_{\mu}^{(g, T)}\right\}$, where $\Pi_{\mu}^{(g, T)}$ is the $g\left(\xi_{T}\right) \exp \left\{\int_{0}^{T} \beta\left(\xi_{s}\right) d s\right\}$-transform of the measure $\Pi_{\mu}(\cdot):=\int_{E} \mu(d x) \Pi_{x}(\cdot)$;

- Given $\left\{\left(\xi_{t}\right)_{0 \leq t \leq T} ; \dot{\mathbf{P}}_{\mu}^{(g, T)}\right\}$, the immigration measure $\left\{\mathbf{n}_{T} ; \dot{\mathbf{P}}_{\mu}^{(g, T)}\left[\cdot \mid\left(\xi_{t}\right)_{0 \leq t \leq T}\right]\right\}$ is a Poisson random measure on $[0, T] \times \mathbb{W}$ with intensity

$$
\mathbf{m}_{T}^{\xi}(d s, d w):=2 \alpha\left(\xi_{s}\right) d s \cdot \mathbb{N}_{\xi_{s}}(d w)+d s \cdot \int_{(0, \infty)} y \mathbf{P}_{y \delta_{\xi_{s}}}(X \in d w) \pi\left(\xi_{s}, d y\right)
$$


- $\left\{\left(Y_{t}\right)_{0 \leq t \leq T} ; \dot{\mathbf{P}}_{\mu}^{(g, T)}\right\}$ is an $\mathcal{M}_{E}^{1}$-valued process defined by

$$
Y_{t}:=\int_{(0, t] \times \mathbb{W}} w_{t-s} \mathbf{n}_{T}(d s, d w), \quad 0 \leq t \leq T .
$$

Theorem 2.8 (Spine representation, [25]). Let $\left\{\left(Y_{t}\right)_{0 \leq t \leq T} ; \dot{\mathbf{P}}_{\mu}^{(g, T)}\right\}$ be the spine representation of $\mathbb{N}_{\mu}^{W_{T}(g)}$ defined above. Then we have

$$
\left\{\left(Y_{t}\right)_{0 \leq t \leq T} ; \dot{\mathbf{P}}_{\mu}^{(g, T)}\right\} \stackrel{\text { f.d.d. }}{=}\left\{\left(W_{t}\right)_{0 \leq t \leq T} ; \mathbb{N}_{\mu}^{W_{T}(g)}\right\} .
$$

Notice that $\mathbf{P}_{\mu}^{X_{T}(g)}\left(X_{0}=\mu\right)=1$. Also notice that $\mathbb{N}_{\mu}$ is not a probability measure, but after the size-biased transform, $\mathbb{N}_{\mu}^{W_{T}(g)}$ is a probability measure. Since $\mathbb{N}_{\mu}\left(\left\|W_{0}\right\| \neq 0\right)=0$, we have $\mathbb{N}_{\mu}^{W_{T}(g)}\left(\left\|W_{0}\right\|=0\right)=1$. Similarly, $\Pi_{\mu}$ is not typically a probability measure, but after the size-biased transform, $\Pi_{\mu}^{(T, g)}$ is a probability measure. We note that

$$
\begin{aligned}
\Pi_{\mu}^{(T, g)}\left[f\left(\xi_{0}\right)\right] & =\frac{1}{\mu\left(P_{T}^{\beta} g\right)} \Pi_{\mu}\left[g\left(\xi_{T}\right) \exp \left\{\int_{0}^{T} \beta\left(\xi_{s}\right) d s\right\} f\left(\xi_{0}\right)\right] \\
& =\frac{1}{\mu\left(P_{T}^{\beta} g\right)} \int_{E}\left(P_{T}^{\beta} g\right)(x) \cdot f(x) \mu(d x)
\end{aligned}
$$

which says that

$$
\Pi_{\mu}^{(T, g)}\left(\xi_{0} \in d x\right)=\frac{1}{\mu\left(P_{T}^{\beta} g\right)}\left(P_{T}^{\beta} g\right)(x) \mu(d x), \quad x \in E .
$$

Now, suppose that $\{\xi ; \Pi\}$ satisfies Assumption 11. Recall that $\phi$ is the principal eigenfunction of the mean semigroup of $X$. The classical spine decomposition theorem, see [6, 7, 21] for example, considered the case $g=\phi$ only. In this case, the family of probabilities $\left(\Pi_{\mu}^{(\phi, T)}\right)_{T \geq 0}$ is consistent in the sense of Kolmogorov's extension theorem, that is, the process $\left\{\left(\xi_{t}\right)_{0 \leq t \leq T} ; \Pi_{\mu}^{(\phi, T)}\right\}$ can be realized as the restriction of some process, say $\left\{\left(\xi_{t}\right)_{t \geq 0} ; \Pi_{\mu}^{(\phi)}\right\}$, on the finite time interval $[0, T]$. In fact, one can also check that this consistency property is satisfied by $\left(\mathbf{P}_{\mu}^{X_{T}(\phi)}\right)_{T \geq 0},\left(\mathbb{N}_{\mu}^{W_{T}(\phi)}\right)_{T \geq 0}$ and $\left(\dot{\mathbf{P}}_{\mu}^{(\phi, T)}\right)_{T \geq 0}$. Therefore, the actual statement of the classical spine decomposition theorem is different from merely replacing $g$ with $\phi$ in Theorem 2.7 and 2.8: There is no need to restrict the corresponding processes on the finite time interval $[0, T]$. Because of its theoretical importance, we state the classical spine decomposition theorem explicitly here:

Corollary 2.9. For each $\mu \in \mathcal{M}_{E}^{\phi} \cap \mathcal{M}_{E}^{1}$, we have

$$
\left\{\left(X_{t}\right)_{t \geq 0} ; \mathbf{P}_{\mu}^{(\phi)}\right\} \stackrel{f . d . d .}{=}\left\{\left(X_{t}+W_{t}\right)_{t \geq 0} ; \mathbf{P}_{\mu} \otimes \mathbb{N}_{\mu}^{(\phi)}\right\} .
$$

Here, the probability $\mathbf{P}_{\mu}^{(\phi)}$ is Doob's h-transform of $\mathbf{P}_{\mu}$ whose restriction on the natural filtration $\left(\mathscr{F}_{t}^{X}\right)$ of the process $\left(X_{t}\right)_{t \geq 0}$ is

$$
d\left(\left.\mathbf{P}_{\mu}^{(\phi)}\right|_{\mathscr{F}_{t}^{X}}\right)=\frac{X_{t}(\phi)}{\mu(\phi)} d\left(\left.\mathbf{P}_{\mu}\right|_{\mathscr{F}_{t}^{X}}\right), \quad t \geq 0
$$


and $\mathbb{N}_{\mu}^{(\phi)}$ is a probability measure on $\mathbb{W}$ whose restriction on the natural filtration $\left(\mathscr{F}_{t}^{W}\right)$ of the process $\left(W_{t}\right)_{t \geq 0}$ is

$$
d\left(\left.\mathbb{N}_{\mu}^{(\phi)}\right|_{\mathscr{F}_{t}^{W}}\right)=\frac{W_{t}(\phi)}{\mu(\phi)} d\left(\left.\mathbb{N}_{\mu}\right|_{\mathscr{F}_{t}^{W}}\right), \quad t \geq 0
$$

Let $\mu \in \mathcal{M}_{\mu}^{(\phi)}$, we say $\left\{\left(\xi_{t}\right)_{t \geq 0}, \mathbf{n},\left(Y_{t}\right)_{t \geq 0} ; \dot{\mathbf{P}}_{\mu}^{(\phi)}\right\}$ is a spine representation of $\mathbb{N}_{\mu}^{(\phi)}$ if:

- The spine process $\left\{\left(\xi_{t}\right)_{t \geq 0} ; \dot{\mathbf{P}}_{\mu}^{(\phi)}\right\}$ is a copy of $\left\{\left(\xi_{t}\right)_{t \geq 0} ; \Pi_{\mu}^{(\phi)}\right\}$ where the probability $\Pi_{\mu}^{(\phi)}$ is Doob's $h$-transform of $\Pi_{\mu}$ whose restriction on the natural filtration $\left(\mathscr{F}_{t}^{\xi}\right)$ of the process $\left(\xi_{t}\right)_{t \geq 0}$ is

$$
d\left(\left.\Pi_{\mu}^{(\phi)}\right|_{\mathscr{F}_{t}^{\xi}}\right)=\frac{\phi\left(\xi_{t}\right) e^{\int_{0}^{t} \beta\left(\xi_{s}\right) d s}}{\mu(\phi)} d\left(\left.\Pi_{\mu}\right|_{\mathscr{F}_{t}^{\xi}}\right), \quad t \geq 0 ;
$$

- Conditioned on $\left\{\left(\xi_{t}\right)_{t \geq 0} ; \dot{\mathbf{P}}_{\mu}^{(\phi)}\right\}$, the immigration measure $\left\{\mathbf{n} ; \dot{\mathbf{P}}_{\mu}^{(\phi)}\left[\cdot \mid\left(\xi_{t}\right)_{t \geq 0}\right]\right\}$ is a Poisson random measure on $[0, \infty) \times \mathbb{W}$ with intensity

$$
\mathbf{m}^{\xi}(d s, d w):=2 \alpha\left(\xi_{s}\right) d s \cdot \mathbb{N}_{\xi_{s}}(d w)+d s \cdot \int_{(0, \infty)} y \mathbf{P}_{y \delta_{\xi_{s}}}(X \in d w) \pi\left(\xi_{s}, d y\right)
$$

- $\left\{\left(Y_{t}\right)_{t \geq 0} ; \dot{\mathbf{P}}_{\mu}^{(\phi)}\right\}$ is an $\mathcal{M}_{E}^{1}$-valued process defined by

$$
Y_{t}:=\int_{(0, t] \times \mathbb{W}} w_{t-s} \mathbf{n}(d s, d w), \quad t \geq 0 .
$$

Corollary 2.10. Let $\left\{\left(Y_{t}\right)_{t \geq 0} ; \dot{\mathbf{P}}_{\mu}^{(\phi)}\right\}$ be the spine representation of $\mathbb{N}_{\mu}^{(\phi)}$ defined above. Then we have

$$
\left\{\left(Y_{t}\right)_{t \geq 0} ; \dot{\mathbf{P}}_{\mu}^{(\phi)}\right\} \stackrel{\text { f.d.d. }}{=}\left\{\left(W_{t}\right)_{t \geq 0} ; \mathbb{N}_{\mu}^{(\phi)}\right\} .
$$

For the sake of generality, the spine decomposition theorems above are all stated with respect to a general initial configuration $\mu$. If $\mu=\delta_{x}$ for some $x \in E$, then by (2.9), we have $\Pi_{\delta_{x}}^{(T, g)}\left(\xi_{0}=x\right)=1$, so sometimes we write $\Pi_{x}^{(T, g)}$ for $\Pi_{\delta_{x}}^{(T, g)}$. Similarly, we write $\Pi_{x}^{(\phi)}$ for $\Pi_{\delta_{x}}^{(\phi)}$.

2.5. Ergodicity of the spine process. In this subsection, we discuss the ergodicity of the spine process $\left\{\left(\xi_{t}\right)_{t \geq 0} ;\left(\Pi_{x}^{(\phi)}\right)_{x \in E}\right\}$ under Assumptions 1 13, According to [14], $\left\{\xi ; \Pi_{x}^{(\phi)}\right\}$ is a time homogeneous Hunt process and its transition density with respect to the measure $m$ is

$$
q_{t}(x, y):=\frac{\phi(y)}{\phi(x)} p_{t}^{\beta}(x, y), \quad x, y \in E, t>0
$$

Let $c_{0}>0$ and $c_{1}>0$ be the constants in (1.21), then we have

$$
\sup _{x \in E}\left|\frac{q_{t}(x, y)}{\phi(y) \phi^{*}(y)}-1\right| \leq c_{0} e^{-c_{1} t}, \quad t>1 \text {. }
$$

This implies that the process $\left\{\xi ; \Pi_{x}^{(\phi)}\right\}$ is ergodic. One can easily get from (2.10) that $\left(\phi \phi^{*}\right)(x) m(d x)$ is the unique invariant probability measure of $\left\{\xi ; \Pi_{x}^{(\phi)}\right\}$. The following 
two lemmas are also simple consequences of (2.10). They will be needed in the proof of Theorem [1.1](3).

Lemma 2.11 ([25, Lemma 5.1]). If $F \in \mathscr{B}_{b}(E \times[0,1] \times[0, \infty)$ is such that $F(y, u):=$ $\lim _{t \rightarrow \infty} F(y, u, t)$ exists for each $y \in E$ and $u \in[0,1]$, then

$$
\int_{0}^{1} F\left(\xi_{(1-u) t}, u, t\right) d u \stackrel{L^{2}\left(\Pi_{x}^{(\phi)}\right)}{\underset{t \rightarrow \infty}{\longrightarrow}} \int_{0}^{1}\left\langle F(\cdot, u), \phi \phi^{*}\right\rangle_{m} d u, \quad x \in E
$$

Lemma 2.12. Let $F \in \mathscr{B}_{b}^{+}(E \times[0,1] \times[0, \infty))$. Define $F(y, u):=\lim _{\sup _{t \rightarrow \infty}} F(y, u, t)$ for each $y \in E$ and $u \in[0,1]$. Then, for each $x \in E$ and $p \geq 1$,

$$
\limsup _{t \rightarrow \infty}\left\|\int_{0}^{1} F\left(\xi_{(1-u) t}, u, t\right) d u\right\|_{\Pi_{x}^{(\phi)} ; L^{p}} \leq \int_{0}^{1}\left\langle F(\cdot, u), \phi \phi^{*}\right\rangle_{m} d u, \quad x \in E .
$$

Proof. For each $(y, u, t) \in E \times[0,1] \times[0, \infty)$, define $\bar{F}(y, u, t):=\sup _{s: s \geq t} F(y, u, s)$. Then $\bar{F} \in \mathscr{B}_{b}(E \times[0,1] \times[0, \infty))$ and

$$
F(x, u)=\lim _{t \rightarrow \infty} \bar{F}(x, u, t), \quad x \in E, u \in[0,1] .
$$

From Lemma 2.11, we know that

$$
\int_{0}^{1} \bar{F}\left(\xi_{(1-u) t}, u, t\right) d u \stackrel{L^{2}\left(\Pi_{x}^{(\phi)}\right)}{\underset{t \rightarrow \infty}{\longrightarrow}} \int_{0}^{1}\left\langle F(\cdot, u), \phi \phi^{*}\right\rangle_{m} d u, \quad x \in E
$$

which implies convergence in probability. The bounded convergence theorem then gives that, for each $p \geq 1$,

$$
\int_{0}^{1} \bar{F}\left(\xi_{(1-u) t}, u, t\right) d u \stackrel{L^{p}\left(\Pi_{x}^{(\phi)}\right)}{\underset{t \rightarrow \infty}{\longrightarrow}} \int_{0}^{1}\left\langle F(\cdot, u), \phi \phi^{*}\right\rangle_{m} d u, \quad x \in E .
$$

Finally, noting that $0 \leq F \leq \bar{F}$, we get

$$
\begin{aligned}
\limsup _{t \rightarrow \infty}\left\|\int_{0}^{1} F\left(\xi_{(1-u) t}, u, t\right) d u\right\|_{\Pi_{x}^{(\phi)} ; L^{p}} & \leq \limsup _{t \rightarrow \infty}\left\|\int_{0}^{1} \bar{F}\left(\xi_{(1-u) t}, u, t\right) d u\right\|_{\Pi_{x}^{(\phi)} ; L^{p}} \\
& =\int_{0}^{1}\left\langle F(\cdot, u), \phi \phi^{*}\right\rangle_{m} d u, \quad x \in E .
\end{aligned}
$$

\section{Proofs}

3.1. Proof of Theorem 1.1(1). Let $\{X ; \mathbf{P}\}$ be a $(\xi, \psi)$-superprocess satisfying Assumptions 14. In this subsection, we will prove the following result stronger than nonpersistency:

Proposition 3.1. For each $t>0, \inf _{x \in E} \mathbf{P}_{\delta_{x}}\left(\left\|X_{t}\right\|=0\right)>0$.

Proof. Recall that $\kappa_{0}=\operatorname{essinf}_{m(d x)} \kappa(x)$ and $\gamma_{0}=\operatorname{essinf}_{m(d x)} \gamma(x)$. For each $x \in E$, let $\widetilde{\kappa}(x):=\kappa(x) \mathbf{1}_{\kappa(x) \geq \kappa_{0}}+\kappa_{0} \mathbf{1}_{\kappa(x)<\kappa_{0}}$ and $\widetilde{\gamma}(x):=\gamma(x) \mathbf{1}_{\gamma(x) \geq \gamma_{0}}+\gamma_{0} \mathbf{1}_{\gamma(x)<\gamma_{0}}$. Then, we know that $m(\widetilde{\kappa} \neq \kappa)=0$ and $m(\widetilde{\gamma} \neq \gamma)=0$. Define $\widetilde{\psi}(x, z):=-\beta(x) z+\widetilde{\kappa}(x) z^{\widetilde{\gamma}(x)}$ for each $x \in E$ and $z \geq 0$, then for each $z \geq 0, \widetilde{\psi}(\cdot, z)=\psi(\cdot, z), m$-almost everywhere. 
If we replace $\psi$ with $\widetilde{\psi}$ in (1.18), the solution $V_{t} f(x)$ of equation (1.18) is also the solution of

$$
V_{t} f(x)+\Pi_{x}\left[\int_{0}^{t \wedge \zeta} \widetilde{\psi}\left(\xi_{s}, V_{t-s} f\right) d s\right]=\Pi_{x}\left[f\left(\xi_{t}\right) \mathbf{1}_{t<\zeta}\right]
$$

So, we can consider $\{X ; \mathbf{P}\}$ as a superprocess with branching mechanism $\widetilde{\psi}$. Define

$$
\widehat{\psi}(z):=-\left(\|\beta\|_{\infty}+\kappa_{0}\right) z+\kappa_{0} z^{\gamma_{0}}, \quad z \geq 0 .
$$

Using the fact that $\gamma_{0}>1$ and $\kappa_{0}>0$, it is easy to verify that

$$
\inf _{x \in E} \widetilde{\psi}(x, z) \geq \widehat{\psi}(z), \quad z \geq 0 ; \quad \int_{1}^{\infty} \frac{1}{\widehat{\psi}(z)} d z<\infty ; \quad \widehat{\psi}(+\infty)=+\infty .
$$

Therefore $\widetilde{\psi}$ satisfies the condition of [26, Lemma 2.3]. As a consequence, we have the desired result.

\subsection{Proof of Theorem 1.1(2).}

Proof of Theorem 1.1(2). Let $\{X ; \mathbf{P}\}$ be a $(\xi, \psi)$-superprocess satisfying Assumptions 14. From Proposition 3.1, we know that

$$
\inf _{x \in E} \mathbf{P}_{\delta_{x}}\left(\left\|X_{t}\right\|=0\right)>0,
$$

which implies that $\{X ; \mathbf{P}\}$ is non-persistent. According to (1.19), Assumption 2 and the fact that $\phi$ is the principal eigenfunction of the semigroup $\left(P_{t}^{\beta}\right)_{t \geq 0}$, we have $\mathbf{P}_{\delta_{x}}\left[X_{t}(\phi)\right]=$ $P_{t}^{\beta} \phi(x)=e^{\lambda t} \phi(x)=\phi(x)>0$. Therefore,

$$
\mathbf{P}_{\delta_{x}}\left(\left\|X_{t}\right\|=0\right)<1, \quad t>0, x \in E .
$$

From (3.1), (3.2) and (2.6), we have that $v_{t} \in \mathscr{B}_{b}^{++}(E)$ for each $t>0$.

According to (2.6) and (2.3), by monotonicity, we see that $\left(v_{t}\right)_{t>0}$ satisfies the equation

$$
v_{s+t}(x)+\int_{0}^{t} P_{t-r}^{\beta} \psi_{0}\left(x, v_{s+r}\right) d r=P_{t}^{\beta} v_{s}(x) \in[0, \infty), \quad s>0, t \geq 0, x \in E .
$$

Notice that, under Assumption 1, according to (1.20), $d \nu:=\phi^{*} d m$ defines a finite measure on $E$. Therefore, $\left\langle v_{t}, \phi^{*}\right\rangle_{m}<\infty$ for each $t>0$. According to (2.4), (2.6) and the monotone convergence theorem, $\left(v_{t}\right)_{t>0}$ also satisfies the equation

$$
\left\langle v_{t}, \phi^{*}\right\rangle_{m}+\int_{s}^{t}\left\langle\psi_{0}\left(\cdot, v_{t}\right), \phi^{*}\right\rangle_{m} d r=\left\langle v_{s}, \phi^{*}\right\rangle_{m} \in[0, \infty), \quad s, t>0 .
$$

One of the consequences of this equation is that, see [25, Lemma 5.2] for example,

$$
\left\|\phi^{-1} v_{t}\right\|_{\infty} \underset{t \rightarrow \infty}{\longrightarrow} 0 .
$$

However, to prove Theorem 1.1(2), we need to consider the speed of this convergence. This is answered in the following two propositions whose proofs are postponed after this proof. The first proposition says that $\left(\phi^{-1} v_{t}\right)(x)$ will converge to 0 with the same speed as $\left\langle v_{t}, \phi^{*}\right\rangle_{m}$, uniformly in $x \in E$ : 
Proposition 3.2. $\left(\phi^{-1} v_{t}\right)(x) \underset{t \rightarrow \infty}{\stackrel{x \in E}{\sim}}\left\langle v_{t}, \phi^{*}\right\rangle_{m}$.

The second proposition characterizes this speed:

Proposition 3.3. $\left(\left\langle v_{t}, \phi^{*}\right\rangle_{m}\right)_{t>0}$ is regularly varying at $\infty$ with index $-\frac{1}{\gamma_{0}-1}$. Furthermore, if $m\left(x: \gamma(x)=\gamma_{0}\right)>0$, then

$$
\left\langle v_{t}, \phi^{*}\right\rangle_{m} \underset{t \rightarrow \infty}{\sim}\left(C_{X}\left(\gamma_{0}-1\right) t\right)^{-\frac{1}{\gamma_{0}-1}}
$$

where $C_{X}:=\left\langle\mathbf{1}_{\gamma=\gamma_{0}} \kappa \phi^{\gamma_{0}}, \phi^{*}\right\rangle_{m}$.

It follows from (2.7) and (3.4) that

$$
-\log \mathbf{P}_{\mu}\left(\left\|X_{t}\right\|=0\right)=\mu\left(v_{t}\right) \leq \mu(\phi)\left\|\phi^{-1} v_{t}\right\|_{\infty} \underset{t \rightarrow \infty}{\longrightarrow} 0 .
$$

Therefore, since $-\log (1-x) \rightarrow 0$ implies $x \rightarrow 0$, we have $\mathbf{P}_{\mu}\left(\left\|X_{t}\right\| \neq 0\right) \underset{t \rightarrow \infty}{\longrightarrow} 0$.

It follows from the fact that $x \underset{x \rightarrow 0}{\sim}-\log (1-x)$, (2.7), Lemma 2.1 and Proposition 3.2 that

$$
\mathbf{P}_{\mu}\left(\left\|X_{t}\right\| \neq 0\right) \underset{t \rightarrow \infty}{\sim}-\log \mathbf{P}_{\mu}\left(\left\|X_{t}\right\|=0\right)=\mu\left(\phi \phi^{-1} v_{t}\right) \underset{t \rightarrow \infty}{\sim} \mu(\phi)\left\langle v_{t}, \phi^{*}\right\rangle_{m}
$$

Then the desired result follows immediately from Proposition 3.3 ,

Proof of Proposition [3.2. We use an argument similar to that used in [25] for critical superprocesses with finite 2 nd moment. We only need to prove that there exists a map $t \mapsto a_{t}>0$ such that

$$
\sup _{x \in E}\left|\frac{\left(\phi^{-1} v_{t}\right)(x)}{a_{t}}-1\right| \underset{t \rightarrow \infty}{\longrightarrow} 0 \text {. }
$$

In fact, once this is proved, we will have that

$$
\begin{aligned}
\left|\frac{\left\langle v_{t}, \phi^{*}\right\rangle_{m}}{a_{t}}-1\right| & \leq \int\left|\frac{\left(\phi^{-1} v_{t}\right)(x)}{a_{t}}-1\right| \phi \phi^{*}(x) m(d x) \\
& \leq \sup _{x \in E}\left|\frac{\left(\phi^{-1} v_{t}\right)(x)}{a_{t}}-1\right| \underset{t \rightarrow \infty}{\longrightarrow} 0 .
\end{aligned}
$$

Then, by (3.5), (3.6) and the property of uniform convergence, we will get the desired result:

$$
\sup _{x \in E}\left|\frac{\left(\phi^{-1} v_{t}\right)(x)}{\left\langle v_{t}, \phi^{*}\right\rangle_{m}}-1\right| \underset{t \rightarrow \infty}{\longrightarrow} 0 .
$$

For each $\mu \in \mathcal{M}_{E}^{\phi}$, denote by $\left\{\left(Y_{t}\right),\left(\xi_{t}\right), \mathbf{n} ; \dot{\mathbf{P}}_{\mu}^{(\phi)}\right\}$ the spine representation of $\mathbb{N}_{\mu}^{(\phi)}$. According to (2.7), (2.8) and Theorem 2.8, we have that for each $t>0$,

$$
\langle\mu, \phi\rangle \dot{\mathbf{P}}_{\mu}^{(\phi)}\left[Y_{t}(\phi)^{-1}\right]=\mathbb{N}_{\mu}\left[W_{t}(\phi)\right] \mathbb{N}_{\mu}^{W_{t}(\phi)}\left[W_{t}(\phi)^{-1}\right]=\mathbb{N}_{\mu}\left(W_{t}(\phi)>0\right)=\mu\left(v_{t}\right) .
$$

Taking $\mu=\delta_{x}$ in (3.7), we get $\left(\phi^{-1} v_{t}\right)(x)=\dot{\mathbf{P}}_{\delta_{x}}^{(\phi)}\left[Y_{t}(\phi)^{-1}\right]$. Recall that $d \nu=\phi^{*} d m$. Taking $\mu=\nu$ in (3.7), we get $\left\langle v_{t}, \phi^{*}\right\rangle_{m}=\dot{\mathbf{P}}_{\nu}^{(\phi)}\left[Y_{t}(\phi)^{-1}\right]$. 
In order to construct an $\left(a_{t}\right)_{t \geq 0}$ satisfying (3.5), we consider a decomposition of the immigration process $\left(Y_{t}\right)_{t \geq 0}$. For any $t>0$ and any $G \in \mathscr{B}((0, t])$, define

$$
Y_{t}^{G}:=\int_{G \times \mathbb{W}} w_{t-s} \mathbf{n}(d s, d w) .
$$

Then for any $0<t_{0}<t$, we can decompose $Y_{t}$ into

$$
Y_{t}=Y_{t}^{\left(0, t_{0}\right]}+Y_{t}^{\left(t_{0}, t\right]} \text {. }
$$

Using this decomposition, for each $0<t_{0}<t<\infty$ and $x \in E$, we have

$$
\dot{\mathbf{P}}_{\delta_{x}}^{(\phi)}\left[Y_{t}(\phi)^{-1}\right]=\dot{\mathbf{P}}_{\nu}^{(\phi)}\left[Y_{t}^{\left(t_{0}, t\right]}(\phi)^{-1}\right]+\epsilon_{x}^{1}\left(t_{0}, t\right)+\epsilon_{x}^{2}\left(t_{0}, t\right),
$$

where

$$
\begin{aligned}
\epsilon_{x}^{1}\left(t_{0}, t\right) & :=\dot{\mathbf{P}}_{\delta_{x}}^{(\phi)}\left[Y_{t}^{\left(t_{0}, t\right]}(\phi)^{-1}\right]-\dot{\mathbf{P}}_{\nu}^{(\phi)}\left[Y_{t}^{\left(t_{0}, t\right]}(\phi)^{-1}\right] ; \\
\epsilon_{x}^{2}\left(t_{0}, t\right) & :=\dot{\mathbf{P}}_{\delta_{x}}^{(\phi)}\left[Y_{t}(\phi)^{-1}-Y_{t}^{\left(t_{0}, t\right]}(\phi)^{-1}\right] .
\end{aligned}
$$

By the construction of the spine representation $\left\{\left(Y_{t}\right),\left(\xi_{t}\right), \mathbf{n} ; \dot{\mathbf{P}}_{\mu}^{(\phi)}\right\}$ and its Markov property, we have that

$$
\begin{aligned}
& \dot{\mathbf{P}}^{(\phi)}\left[Y_{t}^{\left(t_{0}, t\right]}(\phi)^{-1} \mid \mathscr{F}_{t_{0}}^{\xi}\right]=\dot{\mathbf{P}}_{\delta_{\xi_{t_{0}}}^{(\phi)}}^{\left(Y_{t-t_{0}}(\phi)^{-1}\right]}=\left(\phi^{-1} v_{t-t_{0}}\right)\left(\xi_{t_{0}}\right) ; \\
& \dot{\mathbf{P}}_{\nu}^{(\phi)}\left[Y_{t}^{\left(t_{0}, t\right]}(\phi)^{-1}\right]=\Pi_{\nu}^{(\phi)}\left[\left(\phi^{-1} v_{t-t_{0}}\right)\left(\xi_{t_{0}}\right)\right]=\left\langle v_{t-t_{0}}, \phi^{*}\right\rangle_{m} ; \\
& \dot{\mathbf{P}}_{\delta_{x}}^{(\phi)}\left[Y_{t}^{\left(t_{0}, t\right]}(\phi)^{-1}\right]=\Pi_{x}^{(\phi)}\left[\left(\phi^{-1} v_{t-t_{0}}\right)\left(\xi_{t_{0}}\right)\right]=\int_{E} q_{t_{0}}(x, y)\left(\phi^{-1} v_{t-t_{0}}\right)(y) m(d y) .
\end{aligned}
$$

We will show that both $\epsilon_{x}^{1}\left(t_{0}, t\right)$ and $\epsilon_{x}^{2}\left(t_{0}, t\right)$ are very small compared to $\dot{\mathbf{P}}_{\nu}^{(\phi)}\left[Y_{t}^{\left(t_{0}, t\right]}(\phi)^{-1}\right]$ provided $t_{0}$ and $t-t_{0}$ are large enough. This is done in the following two lemmas whose proofs are postponed after this proof.

Let $c_{0}, c_{1}>0$ be the constants in (1.21).

Lemma 3.4. For each $t>t_{0}>1$, we have that

$$
\left|\epsilon_{x}^{1}\left(t_{0}, t\right)\right| \leq c_{0} e^{-c_{1} t_{0}}\left\langle v_{t-t_{0}}, \phi^{*}\right\rangle_{m} .
$$

Lemma 3.5. For each $t_{0}>1$ and $t-t_{0}$ large enough, we have

$$
\left|\epsilon_{x}^{2}\left(t_{0}, t\right)\right| \leq t_{0}\left\|\kappa \gamma \phi^{\gamma-1}\right\|_{\infty} \cdot\left\|\phi^{-1} v_{t-t_{0}}\right\|_{\infty}^{\gamma_{0}-1}\left(1+c_{0} e^{-c_{1} t_{0}}\right)\left\langle v_{t-t_{0}}, \phi^{*}\right\rangle_{m}
$$

Now, for each $t_{0}>1$ and $t-t_{0}$ large enough, according to (3.7), (3.8), (3.9), Lemmas 3.4 and 3.5, we have

$$
\begin{aligned}
& \left|\frac{\left(\phi^{-1} v_{t}\right)(x)}{\left\langle v_{t-t_{0}}, \phi^{*}\right\rangle_{m}}-1\right| \leq \frac{\left|\epsilon_{x}^{1}\left(t_{0}, t\right)\right|}{\left\langle v_{t-t_{0}}, \phi^{*}\right\rangle_{m}}+\frac{\left|\epsilon_{x}^{2}\left(t_{0}, t\right)\right|}{\left\langle v_{t-t_{0}}, \phi^{*}\right\rangle_{m}} \\
& \quad \leq c_{0} e^{-c_{1} t_{0}}+t_{0}\left\|\kappa \gamma \phi^{\gamma-1}\right\|_{\infty} \cdot\left\|\phi^{-1} v_{t-t_{0}}\right\|_{\infty}^{\gamma_{0}-1}\left(1+c_{0} e^{-c_{1} t_{0}}\right) .
\end{aligned}
$$

According to (3.4), there exists a map $t \mapsto t_{0}(t)$ such that,

$$
t_{0}(t) \underset{t \rightarrow \infty}{\longrightarrow} \infty ; \quad t_{0}(t)\left\|\phi^{-1} v_{t-t_{0}(t)}\right\|_{\infty}^{\gamma_{0}-1} \underset{t \rightarrow \infty}{\longrightarrow} 0 .
$$

Plugging this choice of $t_{0}(t)$ into (3.11) and taking $t \rightarrow \infty$, we get the desired assertion (3.5) with $a_{t}:=\left\langle v_{t-t_{0}(t)}, \phi^{*}\right\rangle_{m}$. 
Proof of Lemma 3.4. Note that $c_{0}, c_{1}>0$ are the constants in (1.21). Then for each $t>t_{0}>1$, we have that

$$
\begin{aligned}
\left|\epsilon_{x}^{1}\left(t_{0}, t\right)\right| & =\left|\dot{\mathbf{P}}_{\delta_{x}}^{(\phi)}\left[Y_{t}^{\left(t_{0}, t\right]}(\phi)^{-1}\right]-\dot{\mathbf{P}}_{\nu}^{(\phi)}\left[Y_{t}^{\left(t_{0}, t\right]}(\phi)^{-1}\right]\right| \\
& =\left|\int_{E} q_{t_{0}}(x, y)\left(\phi^{-1} v_{t-t_{0}}\right)(y) m(d y)-\left\langle v_{t-t_{0}}, \phi^{*}\right\rangle_{m}\right| \\
& \leq \int_{y \in E}\left|q_{t_{0}}(x, y)-\left(\phi \phi^{*}\right)(y)\right|\left(\phi^{-1} v_{t-t_{0}}\right)(y) m(d y) \\
& \leq c_{0} e^{-c_{1} t_{0}}\left\langle v_{t-t_{0}}, \phi^{*}\right\rangle_{m} .
\end{aligned}
$$

Proof of Lemma 3.5. Using the Markov property of the spine process and properties of Poisson random measures, we have

$$
\begin{aligned}
\left|\epsilon_{x}^{2}\left(t_{0}, t\right)\right| & =\left|\dot{\mathbf{P}}_{\delta_{x}}^{(\phi)}\left[Y_{t}(\phi)^{-1}-Y_{t}^{\left(t_{0}, t\right]}(\phi)^{-1}\right]\right| \\
& =\dot{\mathbf{P}}_{\delta_{x}}^{(\phi)}\left[Y_{t}^{\left(0, t_{0}\right]}(\phi) \cdot Y_{t}(\phi)^{-1} \cdot Y_{t}^{\left(t_{0}, t\right]}(\phi)^{-1}\right] \\
& \leq \dot{\mathbf{P}}_{\delta_{x}}^{(\phi)}\left[\mathbf{1}_{Y_{t}^{\left(0, t_{0}\right]}(\phi) \neq 0} \cdot Y_{t}^{\left(t_{0}, t\right]}(\phi)^{-1}\right] \\
& =\dot{\mathbf{P}}_{\delta_{x}}^{(\phi)}\left[\dot{\mathbf{P}}_{\delta_{x}}^{(\phi)}\left[\mathbf{1}_{Y_{t}^{\left(0, t_{0}\right]}(\phi) \neq 0} \mid \mathscr{F}_{t_{0}}^{\xi}\right] \cdot \dot{\mathbf{P}}_{\delta_{x}}^{(\phi)}\left[Y_{t}^{\left(t_{0}, t\right]}(\phi)^{-1} \mid \mathscr{F}_{t_{0}}^{\xi}\right]\right] .
\end{aligned}
$$

Since $\phi^{-1} v_{s}$ converges to 0 uniformly when $s \rightarrow \infty$, we can choose $s_{0}>0$ such that for any $s \geq s_{0}$, we have $\left\|\phi^{-1} v_{s}\right\|_{\infty} \leq 1$. With this $s_{0}>0$, we claim that for each $t-t_{0} \geq s_{0}$ the following holds:

$$
\dot{\mathbf{P}}_{\delta_{x}}^{(\phi)}\left[\mathbf{1}_{\left\|Y_{t}^{\left(0, t_{0}\right]}\right\| \neq 0} \mid \mathscr{F}_{t_{0}}^{\xi}\right] \leq t_{0}\left\|\kappa \gamma \phi^{\gamma-1}\right\|_{\infty} \cdot\left\|\phi^{-1} v_{t-t_{0}}\right\|_{\infty}^{\gamma_{0}-1}
$$

We will verify this claim at the end of this proof.

On the other hand, according to (2.10) and (3.10), we know that

$$
\dot{\mathbf{P}}_{\delta_{x}}^{(\phi)}\left[Y_{t}^{\left(t_{0}, t\right]}(\phi)^{-1}\right] \leq\left(1+c_{0} e^{-c_{1} t_{0}}\right)\left\langle v_{t-t_{0}}, \phi^{*}\right\rangle_{m}
$$

Therefore, from (3.12), (3.13) and (3.14), we get that

$$
\begin{aligned}
& \left|\epsilon_{x}^{2}\left(t_{0}, t\right)\right| \leq t_{0}\left\|\kappa \gamma \phi^{\gamma-1}\right\|_{\infty}\left\|\phi^{-1} v_{t-t_{0}}\right\|_{\infty}^{\gamma_{0}-1} \cdot \dot{\mathbf{P}}_{\delta_{x}}^{(\phi)}\left[Y_{t}^{\left(t_{0}, t\right]}(\phi)^{-1}\right] \\
& \leq t_{0}\left\|\kappa \gamma \phi^{\gamma-1}\right\|_{\infty}\left\|\phi^{-1} v_{t-t_{0}}\right\|_{\infty}^{\gamma_{0}-1} \cdot\left(1+c_{0} e^{-c_{1} t_{0}}\right)\left\langle v_{t-t_{0}}, \phi^{*}\right\rangle_{m}
\end{aligned}
$$

as desired.

We now verify the claim (3.13). Note that, if $t-s>t-t_{0} \geq s_{0}$, using the fact that $v_{t}$ is non-increasing in $t$, we get

$$
\kappa(x) \gamma(x) v_{t-s}(x)^{\gamma(x)-1} \leq\left\|\kappa \gamma \phi^{\gamma-1}\right\|_{\infty} \cdot\left\|\phi^{-1} v_{t-s}\right\|_{\infty}^{\gamma_{0}-1} \leq\left\|\kappa \gamma \phi^{\gamma-1}\right\|_{\infty} \cdot\left\|\phi^{-1} v_{t-t_{0}}\right\|_{\infty}^{\gamma_{0}-1} .
$$

Therefore, using Campbell's formula, (1.22) and the fact that $e^{-x} \geq 1-x$, we have, for $t-t_{0} \geq s_{0}$,

$$
\begin{gathered}
\dot{\mathbf{P}}_{\delta_{x}}^{(\phi)}\left[\mathbf{1}_{\left\|Y_{t}^{\left(0, t_{0}\right]}\right\| \neq 0} \mid \mathscr{F}_{t_{0}}^{\xi}\right] \leq-\log \left(1-\dot{\mathbf{P}}_{\delta_{x}}^{(\phi)}\left[\mathbf{1}_{\left\|Y_{t}^{\left(0, t_{0}\right]}\right\| \neq 0} \mid \mathscr{F}_{t_{0}}^{\xi}\right]\right) \\
=-\log \lim _{\lambda \rightarrow \infty} \dot{\mathbf{P}}_{\delta_{x}}^{(\phi)}\left[e^{-\lambda Y_{t}^{\left(0, t_{0}\right]}\left(\mathbf{1}_{E}\right)} \mid \mathscr{F}_{t_{0}}^{\xi}\right]
\end{gathered}
$$




$$
\begin{aligned}
& =-\log \lim _{\lambda \rightarrow \infty} \exp \left\{-\int_{[0, t] \times \mathbb{W}}\left(1-\exp \left\{-\mathbf{1}_{s \leq t_{0}} w_{t-s}\left(\lambda \mathbf{1}_{E}\right)\right\}\right) \mathbf{m}^{\xi}(d s, d w)\right\} \\
& =\int_{[0, t] \times \mathbb{W}} \mathbf{1}_{s \leq t_{0}} \mathbf{1}_{\left\|w_{t-s}\right\| \neq 0} \mathbf{m}^{\xi}(d s, d w)=\int_{0}^{t_{0}} d s \int_{(0, \infty)} y \mathbf{P}_{y \delta_{\xi_{s}}}\left[\mathbf{1}_{\left\|X_{t-s}\right\| \neq 0}\right] \pi\left(\xi_{s}, d y\right) \\
& =\int_{0}^{t_{0}} d s \int_{(0, \infty)} y\left(1-e^{-y v_{t-s}\left(\xi_{s}\right)}\right) \frac{\kappa\left(\xi_{s}\right) d y}{\Gamma\left(-\gamma\left(\xi_{s}\right)\right) y^{1+\gamma\left(\xi_{s}\right)}}=\int_{0}^{t_{0}}\left(\kappa \gamma v_{t-s}^{\gamma-1}\right)\left(\xi_{s}\right) d s \\
& \leq t_{0}\left\|\kappa \gamma \phi^{\gamma-1}\right\|_{\infty} \cdot\left\|\phi^{-1} v_{t-t_{0}}\right\|_{\infty}^{\gamma_{0}-1} .
\end{aligned}
$$

This ends the verification of the claim (3.13), and thus also completes the proof of Lemma 3.5.

Proof of Proposition 3.3. From (3.3) we know that $\left\langle v_{t}, \phi^{*}\right\rangle_{m}$ is continuous and strictly decreasing in $t \in(0, \infty)$. Since the superprocess $\left(X_{t}\right)_{t \geq 0}$ is right continuous in the weak topology with the null measure as an absorbing state, we have that, for each $\mu \in \mathcal{M}_{E}^{1}$, $\mathbf{P}_{\mu}\left(\left\|X_{t}\right\| \neq 0\right) \underset{t \rightarrow 0}{\longrightarrow}$ 1. Taking $\mu=\nu$, according to (2.7), we have that $\left\langle v_{t}, \phi^{*}\right\rangle_{m} \underset{t \rightarrow 0}{\longrightarrow}+\infty$.

On the other hand, according to (3.4), we have

$$
\left\langle v_{t}, \phi^{*}\right\rangle_{m}=\left\langle\phi^{-1} v_{t} \phi, \phi^{*}\right\rangle_{m} \leq\left\|\phi^{-1} v_{t}\right\|_{\infty} \cdot\left\langle\phi, \phi^{*}\right\rangle_{m} \underset{t \rightarrow \infty}{\longrightarrow} 0 .
$$

Therefore, the map $t \mapsto\left\langle v_{t}, \phi^{*}\right\rangle_{m}$ has an inverse on $(0, \infty)$ which is denoted by

$$
R:(0, \infty) \rightarrow(0, \infty) \text {. }
$$

Now, if we denote by

$$
\epsilon_{t}(x):=\frac{v_{t}(x)}{\left\langle v_{t}, \phi^{*}\right\rangle_{m} \phi(x)}-1, \quad t>0, x \in E
$$

then we have

$$
v_{t}(x)=\left(1+\epsilon_{R\left(\left\langle v_{t}, \phi^{*}\right\rangle_{m}\right)}(x)\right)\left\langle v_{t}, \phi^{*}\right\rangle_{m} \phi(x), \quad t>0, x \in E .
$$

Further, by Proposition 3.2 and the fact that $R(u) \underset{u \rightarrow 0}{\longrightarrow} \infty$, we have

$$
\sup _{x \in E}\left|\epsilon_{R(u)}(x)\right| \underset{u \rightarrow 0}{\longrightarrow} 0 \text {. }
$$

Now, by (3.3), we have

Therefore,

$$
\frac{d\left\langle v_{r}, \phi^{*}\right\rangle_{m}}{d r}=-\left\langle\psi_{0}\left(\cdot, v_{r}\right), \phi^{*}\right\rangle_{m}>0 \quad \text { a.e.. }
$$

$$
\begin{aligned}
s-t & =\int_{t}^{s} d r=\int_{s}^{t}\left\langle\psi_{0}\left(\cdot, v_{r}\right), \phi^{*}\right\rangle_{m}^{-1} d\left\langle v_{r}, \phi^{*}\right\rangle_{m} \\
& \stackrel{\text { by }}{\stackrel{(3.15)}{=}} \int_{s}^{t}\left\langle\psi_{0}\left(\cdot,\left(1+\epsilon_{R\left(\left\langle v_{r}, \phi^{*}\right\rangle_{m}\right)}\right)\left\langle v_{r}, \phi^{*}\right\rangle_{m} \phi\right), \phi^{*}\right\rangle_{m}^{-1} d\left\langle v_{r}, \phi^{*}\right\rangle_{m} \\
& =\int_{\left\langle v_{s}, \phi^{*}\right\rangle}^{\left\langle v_{t}, \phi^{*}\right\rangle}\left\langle\psi_{0}\left(\cdot,\left(1+\epsilon_{R(u)}\right) u \phi\right), \phi^{*}\right\rangle_{m}^{-1} d u .
\end{aligned}
$$


Letting $t \rightarrow 0$, we get

$$
s=\int_{\left\langle v_{s}, \phi^{*}\right\rangle}^{\infty}\left\langle\psi_{0}\left(\cdot,\left(1+\epsilon_{R(u)}\right) u \phi\right), \phi^{*}\right\rangle_{m}^{-1} d u, \quad s \in(0, \infty) .
$$

Since $R$ is the inverse of $t \mapsto\left\langle v_{t}, \phi^{*}\right\rangle$, the above implies that

$$
R(r)=\int_{r}^{\infty}\left\langle\psi_{0}\left(\cdot,\left(1+\epsilon_{R(u)}\right) u \phi\right), \phi^{*}\right\rangle_{m}^{-1} d u, \quad r \in(0, \infty) .
$$

We now check the regularly varying property of $R(r)$ at $r=0$. This can be done by considering the regularly varying property of $u \rightarrow\left\langle\psi_{0}\left(\cdot,\left(1+\epsilon_{R(u)}\right) u \phi\right), \phi^{*}\right\rangle_{m}$ at 0 . According to (3.16) $), 1+\epsilon_{R(u)}(x) \underset{u \rightarrow 0}{\stackrel{x \in E}{\sim}} 1$. Since $\gamma(\cdot)$ is bounded, we have $\left(1+\epsilon_{R(u)}(x)\right) \underset{u \rightarrow 0}{\gamma(x) \underset{x \in E}{\sim}}$ 1. Therefore, from Lemma 2.1, we have that

$$
\begin{aligned}
& \left\langle\psi_{0}\left(\cdot,\left(1+\epsilon_{R(u)}\right) u \phi\right), \phi^{*}\right\rangle_{m} \\
& \quad=\left\langle\kappa(x)\left(1+\epsilon_{R(u)}(x)\right)^{\gamma(x)} u^{\gamma(x)} \phi(x)^{\gamma(x)}, \phi^{*}(x)\right\rangle_{m(d x)} \\
& \underset{u \rightarrow 0}{\sim}\left\langle u^{\gamma(x)}, \kappa(x) \phi(x)^{\gamma(x)} \phi^{*}(x)\right\rangle_{m(d x)} .
\end{aligned}
$$

According to Lemma 2.6, and using the fact that $\kappa(x) \phi(x)^{\gamma(x)}$ is bounded and the measure $\phi^{*} d m$ is finite, we have that $\left\langle\psi_{0}\left(\cdot,\left(1+\epsilon_{R(u)}\right) u \phi\right), \phi^{*}\right\rangle_{m}$ is regularly varying at $u=0$ with index $\gamma_{0}$. Noticing that $-\left(\gamma_{0}-1\right)<0$, according to Corollary 2.3 and (3.17), $R$ is regularly varying at 0 with index $-\left(\gamma_{0}-1\right)$. Therefore, from $R\left(\left\langle v_{s}, \phi^{*}\right\rangle_{m}\right)=s$ and Corollary 2.5, we have that $\left(\left\langle v_{s}, \phi^{*}\right\rangle_{m}\right)_{s \in(0, \infty)}$ is regularly varying at $\infty$ with index $-\left(\gamma_{0}-1\right)^{-1}$.

Further, if $m\left\{x: \gamma(x)=\gamma_{0}\right\}>0$, then according to Lemma 2.6 and (3.18), we know that

$$
\begin{gathered}
\left\langle\psi_{0}\left(\cdot,\left(1+\epsilon_{R(u)}\right) u \phi\right), \phi^{*}\right\rangle_{m} \underset{u \rightarrow 0}{\sim}\left\langle u^{\gamma(x)}, \kappa(x) \phi(x)^{\gamma(x)} \phi^{*}(x)\right\rangle_{m(d x)} \\
\underset{u \rightarrow 0}{\sim}\left\langle\mathbf{1}_{\gamma(x)=\gamma_{0}}, \kappa(x) \phi(x)^{\gamma_{0}} \phi^{*}(x)\right\rangle_{m(d x)} u^{\gamma_{0}}=: C_{X} u^{\gamma_{0}} .
\end{gathered}
$$

Therefore, we have $\left\langle\psi_{0}\left(\cdot,\left(1+\epsilon_{R(u)}\right) u \phi\right), \phi^{*}\right\rangle_{m}^{-1}=u^{-\gamma_{0}} l(u)$, where $l(u)$ converges to the constant $C_{X}^{-1}$ when $u \rightarrow 0$. Now according to Corollary 2.3 and (3.17) we have that

$$
\begin{aligned}
R(r) & =\int_{r}^{\infty}\left\langle\psi_{0}\left(\cdot,\left(1+\epsilon_{R(u)}\right) u \phi\right), \phi^{*}\right\rangle_{m}^{-1} d u=\int_{r}^{\infty} u^{-\gamma_{0}} l(u) d u \\
& =-\frac{1}{\gamma_{0}-1} \int_{r}^{\infty} l(u) d u^{-\left(\gamma_{0}-1\right)} \\
& \underset{r \rightarrow 0}{\sim} C_{X}^{-1}\left(\gamma_{0}-1\right)^{-1} r^{-\left(\gamma_{0}-1\right)} .
\end{aligned}
$$

Finally since $r \mapsto\left\langle v_{r}, \phi^{*}\right\rangle_{m}$ is the inverse of $r \mapsto R(r)$, from [4, Proposition 1.5.15.] and the above, we have

$$
\left\langle v_{r}, \phi^{*}\right\rangle_{m} \underset{r \rightarrow \infty}{\sim}\left(C_{X}\left(\gamma_{0}-1\right) r\right)^{-\frac{1}{\gamma_{0}-\llbracket}}
$$


3.3. Characterization of the one dimensional distribution. Let $\left\{\left(X_{t}\right)_{t \geq 0} ; \mathbf{P}\right\}$ be a $(\xi, \psi)$-superprocess satisfying Assumptions 114. Suppose $m\left(x: \gamma(x)=\gamma_{0}\right)>0$. Recall that we want to find a proper normalization $\left(\eta_{t}\right)_{t \geq 0}$ such that $\left\{\left(\eta_{t} X_{t}(f)\right)_{t \geq 0} ; \mathbf{P}_{\mu}\left(\cdot \mid\left\|X_{t}\right\| \neq\right.\right.$ $0)\}$ converges weakly to a non-degenerate distribution for a large class of functions $f$ and initial configurations $\mu$. Our guess of $\left(\eta_{t}\right)$ is

$$
\eta_{t}:=\left(C_{X}\left(\gamma_{0}-1\right) t\right)^{-\frac{1}{\gamma_{0}-1}}, \quad t \geq 0
$$

because in this case

$$
\mathbf{P}_{\delta_{x}}\left[\eta_{t} X_{t}(f) \mid\left\|X_{t}\right\| \neq 0\right]=\frac{\mathbf{P}_{\delta_{x}}\left[\eta_{t} X_{t}(f) \mathbf{1}_{\left\|X_{t}\right\| \neq 0}\right]}{\mathbf{P}_{\delta_{x}}\left(\left\|X_{t}\right\| \neq 0\right)}=\frac{\eta_{t}}{\mathbf{P}_{\delta_{x}}\left(\left\|X_{t}\right\| \neq 0\right)} P_{t}^{\beta} f(x) \underset{t \rightarrow \infty}{\sim}\left\langle f, \phi^{*}\right\rangle_{m} .
$$

Here we have used Theorem 1.1(2) and the fact that (see (1.21))

$$
P_{t}^{\beta} f(x)=\int_{E} p_{t}^{\beta}(x, y) f(y) d y \underset{t \rightarrow \infty}{\longrightarrow} \phi(x)\left\langle f, \phi^{*}\right\rangle_{m} .
$$

From the point of view of Laplace transforms, the desired result that, for any $f \in$ $\mathscr{B}_{b}^{+}(E)$ and $\mu \in \mathcal{M}_{E}^{1},\left\{\left(\eta_{t} X_{t}(f)\right)_{t \geq 0} ; \mathbf{P}_{\mu}\left(\cdot \mid\left\|X_{t}\right\| \neq 0\right)\right\}$ converge weakly to some probability distribution $F_{f}$ is equivalent to the following convergence:

$$
\mathbf{P}_{\mu}\left[1-e^{-\theta \eta_{t} X_{t}(f)} \mid\left\|X_{t}\right\| \neq 0\right]=\frac{1-\exp \left\{-\mu\left(V_{t}\left(\theta \eta_{t} f\right)\right)\right\}}{\mathbf{P}_{\mu}\left(\left\|X_{t}\right\| \neq 0\right)} \underset{t \rightarrow \infty}{\longrightarrow} \int_{[0, \infty)}\left(1-e^{-\theta u}\right) F_{f}(d u) .
$$

According to Theorem $1.1(2)$ and $1-e^{-x} \underset{x \rightarrow 0}{\sim} x$, this is equivalent to

$$
\frac{\mu\left(V_{t}\left(\theta \eta_{t} f\right)\right)}{\eta_{t}} \underset{t \rightarrow \infty}{\longrightarrow} \mu(\phi) \int_{[0, \infty)}\left(1-e^{-\theta u}\right) F_{f}(d u) .
$$

Therefore, to establish the weak convergence of $\left\{\left(\eta_{t} X_{t}(f)\right)_{t \geq 0} ; \mathbf{P}_{\mu}\left(\cdot \mid\left\|X_{t}\right\| \neq 0\right)\right\}$, one only needs to verify (3.20).

In order to investigate the convergence of $\mu\left(V_{t}\left(\theta \eta_{t} f\right)\right) / \eta_{t}$, we need to investigate the properties of $\theta \rightarrow V_{t}(\theta f)$. (Note that (2.3) only gives the the dynamics of $t \rightarrow V_{t}(\theta f)$.) This is done in the following proposition:

Proposition 3.6. For any $f \in \mathscr{B}_{b}^{+}(E), \theta \geq 0, x \in E$ and $T>0$, we have

$$
V_{T}(\theta f)(x)=\phi(x) \int_{0}^{\theta} \Pi_{x}^{(\phi)}\left[\frac{f\left(\xi_{T}\right)}{\phi\left(\xi_{T}\right)} \exp \left\{-\int_{0}^{T}\left(\kappa \gamma V_{T-s}(r f)^{\gamma-1}\right)\left(\xi_{s}\right) d s\right\}\right] d r
$$

Proof. It follows from Theorems 2.7 and 2.8 that

$$
\frac{\mathbf{P}_{\delta_{x}}\left[X_{T}(f) e^{-\theta X_{T}(f)}\right]}{\mathbf{P}_{\delta_{x}}\left[X_{T}(f)\right]}=\mathbf{P}_{\delta_{x}}^{X_{T}(f)}\left[e^{-\theta X_{T}(f)}\right]=\mathbf{P}_{\delta_{x}}\left[e^{-\theta X_{T}(f)}\right] \dot{\mathbf{P}}_{x}^{(T, f)}\left[e^{-\theta Y_{T}(f)}\right]
$$

where $\left\{(\xi)_{0 \leq t \leq T}, \mathbf{n}_{T},(Y)_{0 \leq t \leq T} ; \dot{\mathbf{P}}_{x}^{(f, T)}\right\}$ is the spine representation of $\mathbb{N}_{x}^{W_{T}(f)}$ with $\mathbf{m}_{T}^{\xi}$ being the intensity of the immigration measure $\mathbf{n}_{T}$ conditioned on $\left\{(\xi)_{0 \leq t \leq T} ; \dot{\mathbf{P}}_{x}^{(f, T)}\right\}$. From this, we have

$$
\frac{\partial}{\partial \theta}\left(-\log \mathbf{P}_{\delta_{x}}\left[e^{-\theta X_{T}(f)}\right]\right)=\frac{\mathbf{P}_{\delta_{x}}\left[X_{T}(f) e^{-\theta X_{T}(f)}\right]}{\mathbf{P}_{\delta_{x}}\left[e^{-\theta X_{T}(f)}\right]}=P_{T}^{\beta} f(x) \dot{\mathbf{P}}_{x}^{(T, f)}\left[e^{-\theta Y_{T}(f)}\right] .
$$


On the other hand, if we write $F(s, w):=\mathbf{1}_{s \leq T} w_{T-s}(f)$, then by Assumption 4, the spine representation, Campbell's formula and (1.22), we have

$$
\begin{aligned}
- & \log \dot{\mathbf{P}}_{x}^{(T, f)}\left[e^{-\theta \mathbf{n}_{T}(F)} \mid \mathbf{m}_{T}^{\xi}\right]=\mathbf{m}_{T}^{\xi}\left(1-e^{-\theta F}\right) \\
& =\int_{0}^{T} d s \int_{(0, \infty)} y \mathbf{P}_{y \delta_{\xi_{s}}}\left[1-e^{-\theta X_{T-s}(f)}\right] \pi\left(\xi_{s}, y\right) \\
& =\int_{0}^{T} d s \cdot \kappa\left(\xi_{s}\right) \int_{(0, \infty)}\left(1-e^{-y V_{T-s}(\theta f)\left(\xi_{s}\right)}\right) \frac{d y}{\Gamma\left(-\gamma\left(\xi_{s}\right)\right) y^{\gamma\left(\xi_{s}\right)}} \\
& =\int_{0}^{T}\left(\kappa \gamma V_{T-s}(\theta f)^{\gamma-1}\right)\left(\xi_{s}\right) d s .
\end{aligned}
$$

Note that, since $\mathbf{n}_{T}(F)=Y_{T}(f)$, we can derive from (3.22) and (3.23) that

$$
\begin{aligned}
V_{T}(\theta f)(x) & =-\log \mathbf{P}_{\delta_{x}}\left[e^{-\theta X_{T}(f)}\right]=\int_{0}^{\theta} P_{T}^{\beta} f(x) \dot{\mathbf{P}}_{x}^{(T, f)}\left[e^{-r Y_{T}(f)}\right] d r \\
& =P_{T}^{\beta} f(x) \int_{0}^{\theta} \Pi_{x}^{(T, f)}\left[\exp \left\{-\int_{0}^{T}\left(\kappa \gamma V_{T-s}(r f)^{\gamma-1}\right)\left(\xi_{s}\right) d s\right\}\right] d r \\
& =\phi(x) \int_{0}^{\theta} \Pi_{x}^{(\phi)}\left[\frac{f\left(\xi_{T}\right)}{\phi\left(\xi_{T}\right)} \exp \left\{-\int_{0}^{T}\left(\kappa \gamma V_{T-s}(r f)^{\gamma-1}\right)\left(\xi_{s}\right) d s\right\}\right] d r
\end{aligned}
$$

as required.

Replacing $\theta$ with $\theta \eta_{T}$ in (3.21), we have

$$
\begin{aligned}
& (3.24) \frac{V_{T}\left(\theta \eta_{T} f\right)(x)}{\eta_{T}} \\
& =\phi(x) \frac{1}{\eta_{T}} \int_{0}^{\theta \eta_{T}} \Pi_{x}^{(\phi)}\left[\frac{f\left(\xi_{T}\right)}{\phi\left(\xi_{T}\right)} \exp \left\{-\int_{0}^{T}\left(\kappa \gamma V_{T-s}(r f)^{\gamma-1}\right)\left(\xi_{s}\right) d s\right\}\right] d r \\
& =\phi(x) \int_{0}^{\theta} \Pi_{x}^{(\phi)}\left[\frac{f\left(\xi_{T}\right)}{\phi\left(\xi_{T}\right)} \exp \left\{-\int_{0}^{T}\left(\kappa \gamma V_{T-s}\left(r \eta_{T} f\right)^{\gamma-1}\right)\left(\xi_{s}\right) d s\right\}\right] d r \\
& =\phi(x) \int_{0}^{\theta} \Pi_{x}^{(\phi)}\left[\frac{f\left(\xi_{T}\right)}{\phi\left(\xi_{T}\right)} \exp \left\{-T \int_{0}^{1}\left(\kappa \gamma V_{u T}\left(r \eta_{T} f\right)^{\gamma-1}\right)\left(\xi_{(1-u) T}\right) d u\right\}\right] d r .
\end{aligned}
$$

3.4. Proof of Theorem 1.1(3). Consider the $(\xi, \psi)$-superprocess $\{X ; \mathbf{P}\}$ which satisfies Assumptions 1 4. Suppose that $m\left(x: \gamma(x)=\gamma_{0}\right)>0$. Let $f \in \mathscr{B}^{+}(E)$ be such that $\left\langle f, \phi^{*}\right\rangle_{m}>0$ and $c_{f}:=\left\|\phi^{-1} f\right\|_{\infty}<\infty$.

Without loss of generality, we assume that $\left\langle f, \phi^{*}\right\rangle_{m}=1$. We claim that, in order to prove Theorem $1.1(3)$, we only need to show that

$$
g(t, \theta, x):=\frac{V_{t}\left(\theta \eta_{t} f\right)(x)}{\eta_{t} \phi(x)} \underset{t \rightarrow \infty}{\longrightarrow} G(\theta):=\left(\frac{1}{1+\theta^{-\left(\gamma_{0}-1\right)}}\right)^{\frac{1}{\gamma_{0}-1}}, \quad x \in E, \theta \geq 0 .
$$


In fact, by (3.24), we have $\left\|V_{t}\left(\theta \eta_{t} f\right) / \eta_{t}\right\|_{\infty} \leq \theta\|\phi\|_{\infty}\left\|\phi^{-1} f\right\|_{\infty}$. Therefore, if (3.25) is true, then by the bounded convergence theorem, for each $\mu \in \mathcal{M}_{E}^{1}$,

$$
\frac{\mu\left(V_{t}\left(\theta \eta_{t} f\right)\right)}{\eta_{t}} \underset{t \rightarrow \infty}{\longrightarrow} \mu(\phi) G(\theta)
$$

which, by the discussion in Subsection 3.3, is equivalent to Theorem 1.1(3).

From Lemma A.9, we have that $G$ satisfies

$$
G(\theta)=\int_{0}^{\theta} e^{-\frac{1}{\gamma_{0}-1} J_{G}(r)} d r, \quad \theta \geq 0,
$$

where

$$
J_{G}(r):=\gamma_{0} \int_{0}^{1} G\left(r u^{\frac{1}{\gamma_{0}-1}}\right)^{\gamma_{0}-1} \frac{d u}{u}, \quad r \geq 0 .
$$

According to (3.24), we know that $g$ satisfies

$$
g(t, \theta, x)=\int_{0}^{\theta} \Pi_{x}^{(\phi)}\left[\left(\phi^{-1} f\right)\left(\xi_{t}\right) e^{-\frac{1}{\gamma_{0}-1} J_{g}(t, r, \xi)}\right] d r, \quad t \geq 0, \theta \geq 0, x \in E,
$$

where, for each $t \geq 0$ and $r \geq 0$,

$$
J_{g}(t, r, \xi):=\left(\gamma_{0}-1\right) t \int_{0}^{1}\left(\kappa \gamma \cdot\left(\phi \eta_{u t}\right)^{\gamma-1} g\left(u t, r u^{\frac{1}{\gamma_{0}-1}}, \cdot\right)^{\gamma-1}\right)\left(\xi_{(1-u) t}\right) d u .
$$

For each $t \geq 0$ and $r \geq 0$, define

$$
J_{G}^{\prime}(t, r, \xi):=\gamma_{0}\left(\gamma_{0}-1\right) t \int_{0}^{1}\left(\mathbf{1}_{\gamma(\cdot)=\gamma_{0}} \kappa \cdot\left(\phi \eta_{u t}\right)^{\gamma_{0}-1} G\left(r u^{\frac{1}{\gamma_{0}-1}}\right)^{\gamma_{0}-1}\right)\left(\xi_{(1-u) t}\right) d u
$$

and

$$
J_{g}^{\prime}(t, r, \xi):=\gamma_{0}\left(\gamma_{0}-1\right) t \int_{0}^{1}\left(\mathbf{1}_{\gamma(\cdot)=\gamma_{0}} \kappa \cdot\left(\phi \eta_{u t}\right)^{\gamma_{0}-1} g\left(u t, r u^{\frac{1}{\gamma_{0}-1}}, \cdot\right)^{\gamma_{0}-1}\right)\left(\xi_{(1-u) t}\right) d u
$$

The main idea is to show that $J_{G}, J_{G}^{\prime}, J_{g}$ and $J_{g}^{\prime}$ are approximately equal in some sense when $t \rightarrow \infty$.

Step 1: We will give upper bounds for $G, g, J_{G}, J_{G}^{\prime}, J_{g}$ and $J_{g}^{\prime}$ respectively. From (3.26) we have

$$
G(r) \leq r, \quad r \geq 0
$$

From (3.27) and (3.32), we have

$$
J_{G}(r) \leq \gamma_{0} r^{\gamma_{0}-1}, \quad r \geq 0 .
$$

From (3.28), we have

$$
g(t, r, x) \leq c_{f} r, \quad t \geq 0, r \geq 0, x \in E .
$$

From (3.19), (3.29), (3.34) and the fact that $\gamma(\cdot)-1<1$, we have $\Pi^{(\phi)}$-almost surely

$$
J_{g}(t, r, \xi) \leq\left\|\kappa \cdot\left(c_{f} \phi\right)^{\gamma-1}\right\|_{\infty} \int_{0}^{1}\left(t \eta_{u t}^{\gamma-1}\left(r u^{\frac{1}{\gamma_{0}-1}}\right)^{\gamma-1}\right)\left(\xi_{(1-u) t}\right) d u
$$




$$
\begin{aligned}
& =\left\|\kappa \cdot\left(c_{f} \phi\right)^{\gamma-1}\right\|_{\infty} \int_{0}^{1}\left(r^{\gamma-1} t^{1-\frac{\gamma-1}{\gamma_{0}-1}}\left(C_{X}\left(\gamma_{0}-1\right)\right)^{-\frac{\gamma-1}{\gamma_{0}-1}}\right)\left(\xi_{(1-u) t}\right) d u \\
& \leq \max \{1, r\} \cdot\left\|\kappa \cdot\left(c_{f} \phi\right)^{\gamma-1}\right\|_{\infty}\left\|\left(C_{X}\left(\gamma_{0}-1\right)\right)^{-\frac{\gamma-1}{\gamma_{0}-1}}\right\|_{\infty} \\
& =: c_{2} \cdot \max \{1, r\}, \quad t \geq 1, r \geq 0 .
\end{aligned}
$$

From (3.19), (3.31) and (3.34), we have $\Pi^{(\phi)}$-almost surely

$$
\begin{aligned}
J_{g}^{\prime}(t, r, \xi) & \leq \gamma_{0}\left(\gamma_{0}-1\right) t \int_{0}^{1}\left(\mathbf{1}_{\gamma(\cdot)=\gamma_{0}} \kappa \cdot\left(\phi \eta_{u t}\right)^{\gamma_{0}-1}\left(c_{f} r u^{\frac{1}{\gamma_{0}-1}}\right)^{\gamma_{0}-1}\right)\left(\xi_{(1-u) t}\right) d u \\
& \leq \gamma_{0}\left(\gamma_{0}-1\right) c_{f}^{\gamma_{0}-1} r^{\gamma_{0}-1}\left\|\mathbf{1}_{\gamma(\cdot)=\gamma_{0}} \kappa \phi^{\gamma_{0}-1}\right\|_{\infty} \int_{0}^{1} t\left(C_{X}\left(\gamma_{0}-1\right) u t\right)^{-1} u d u \\
& =: c_{3} \cdot r^{\gamma_{0}-1}, \quad t \geq 0, r \geq 0 .
\end{aligned}
$$

From (3.19), (3.30) and (3.32), we have $\Pi^{(\phi)}$-almost surely

$$
\begin{aligned}
J_{G}^{\prime}(t, r, \xi) & \leq \gamma_{0}\left(\gamma_{0}-1\right) t \int_{0}^{1}\left(\mathbf{1}_{\gamma(\cdot)=\gamma_{0}} \kappa \cdot\left(\phi \eta_{u t}\right)^{\gamma_{0}-1}\left(r u^{\frac{1}{\gamma_{0}-1}}\right)^{\gamma_{0}-1}\right)\left(\xi_{(1-u) t}\right) d u \\
& \leq \gamma_{0}\left(\gamma_{0}-1\right) r^{\gamma_{0}-1}\left\|\mathbf{1}_{\gamma(\cdot)=\gamma_{0}} \kappa \phi^{\gamma_{0}-1}\right\|_{\infty} \int_{0}^{1} t\left(C_{X}\left(\gamma_{0}-1\right) u t\right)^{-1} u d u \\
& =: c_{4} \cdot r^{\gamma_{0}-1}, \quad t \geq 0, r \geq 0 .
\end{aligned}
$$

In the remainder of this proof, we use the following notation: If $f$ is a measurable function which is $L^{p}$ integrable on the measure space $(S, \mathscr{S}, \mu)$ with $p>0$, then we write

$$
\|f\|_{\mu ; p}:=\left(\int_{S}|f|^{p} d \mu\right)^{\frac{1}{p}}
$$

Notice that, when $p \geq 1,\|f\|_{\mu ; p}$ is simply the $L^{p}$ norm of $f$ with respect to the measure $\mu$. However, when $p \in(0,1),\|\cdot\|_{\mu ; p}$ is not a norm.

Step 2: We will show that, for each $t \geq 0, \theta \geq 0$, and $x \in E$

$$
\begin{aligned}
& \left|G(\theta)^{\gamma_{0}-1}-g(t, \theta, x)^{\gamma_{0}-1}\right| \\
& \quad \leq I_{1}(t, \theta, x)+c_{f}^{\gamma_{0}-1} I_{2}(t, \theta, x)+c_{f}^{\gamma_{0}-1} I_{3}(t, \theta, x)+c_{f}^{\gamma_{0}-1} I_{4}(t, \theta, x),
\end{aligned}
$$

where

$$
\begin{aligned}
& I_{1}(t, \theta, x):=\left\|e^{-J_{G}(r)}-\right\|\left(\phi^{-1} f\right)\left(\xi_{t}\right)^{\gamma_{0}-1} e^{-J_{G}(r)}\left\|_{\Pi_{x}^{(\phi)} ; \frac{1}{\gamma_{0}-1}}\right\|_{\mathbf{1}_{0 \leq r \leq \theta} d r ; \frac{1}{\gamma_{0}-1}}, \\
& I_{2}(t, \theta, x):=\|\| J_{G}(r)-J_{G}^{\prime}(t, r, \xi)\left\|_{\Pi_{x}^{(\phi)} ; \frac{1}{\gamma_{0}-1}}\right\|_{\mathbf{1}_{0 \leq r \leq \theta} d r ; \frac{1}{\gamma_{0}-1}}, \\
& I_{3}(t, \theta, x):=\|\| J_{G}^{\prime}(t, r, \xi)-J_{g}^{\prime}(t, r, \xi)\left\|_{\Pi_{x}^{(\phi)} ; \frac{1}{\gamma_{0}-1}}\right\|_{\mathbf{1}_{0 \leq r \leq \theta} d r ; \frac{1}{\gamma_{0}-1}},
\end{aligned}
$$

and

$$
I_{4}(t, \theta, x):=\|\| J_{g}^{\prime}(t, r, \xi)-J_{g}(t, r, \xi)\left\|_{\Pi_{x}^{(\phi)} ; \frac{1}{\gamma_{0}-1}}\right\|_{\mathbf{1}_{0 \leq r \leq \theta} d r ; \frac{1}{\gamma_{0}-1}} .
$$


In fact, we can rewrite $(3.26)$ and $(3.28)$ as:

$$
G(\theta)^{\gamma_{0}-1}=\left\|e^{-J_{G}(r)}\right\|_{\mathbf{1}_{0 \leq r \leq \theta} d r ; \frac{1}{\gamma_{0}-1}}, \quad \theta \geq 0,
$$

and

$$
g(t, \theta, x)^{\gamma_{0}-1}=\|\|\left(\phi^{-1} f\right)\left(\xi_{t}\right)^{\gamma_{0}-1} e^{-J_{g}(t, r, \xi)}\left\|_{\Pi_{x}^{(\phi)} ; \frac{1}{\gamma_{0}-1}}\right\|_{\mathbf{1}_{0 \leq r \leq \theta} d r ; \frac{1}{\gamma_{0}-1}}, \quad t \geq 0, \theta \geq 0, x \in E .
$$

Therefore, by Minkowski's inequality we have that, for each $t \geq 0, \theta \geq 0$ and $x \in E$,

$$
\begin{aligned}
& \left|G(\theta)^{\gamma_{0}-1}-g(t, \theta, x)^{\gamma_{0}-1}\right| \\
& \quad \leq\left\|e^{-J_{G}(r)}-\right\|\left(\phi^{-1} f\right)\left(\xi_{t}\right)^{\gamma_{0}-1} e^{-J_{g}(t, r, \xi)}\left\|_{\Pi_{x}^{(\phi)} ; \frac{1}{\gamma_{0}-1}}\right\|_{\mathbf{1}_{0 \leq r \leq \theta} d r ; \frac{1}{\gamma_{0}-1}} \\
& \quad \leq I_{1}(t, \theta, x)+\|\|\left(\phi^{-1} f\right)\left(\xi_{t}\right)^{\gamma_{0}-1} e^{-J_{G}(r)} \|_{\Pi_{x}^{(\phi)} ; \frac{1}{\gamma_{0}-1}}- \\
& \quad\left\|\left(\phi^{-1} f\right)\left(\xi_{t}\right)^{\gamma_{0}-1} e^{-J_{g}(t, r, \xi)}\right\|_{\Pi_{x}^{(\phi)} ; \frac{1}{\gamma_{0}-1}} \|_{\mathbf{1}_{0 \leq r \leq \theta} d r ; \frac{1}{\gamma_{0}-1}} \\
& \quad \leq I_{1}(t, \theta, x)+\|\|\left(\phi^{-1} f\right)\left(\xi_{t}\right)^{\gamma_{0}-1}\left(e^{-J_{G}(r)}-e^{-J_{g}(t, r, \xi)}\right)\left\|_{\Pi_{x}^{(\phi)} ; \frac{1}{\gamma_{0}-1}}\right\|_{\mathbf{1}_{0 \leq r \leq \theta} d r ; \frac{1}{\gamma_{0}-1}} \\
& \quad \leq I_{1}(t, \theta, x)+c_{f}^{\gamma_{0}-1}\|\| J_{G}(r)-J_{g}(t, r, \xi)\left\|_{\Pi_{x}^{(\phi)} ; \frac{1}{\gamma_{0}-1}}\right\|_{\mathbf{1}_{0 \leq r \leq \theta} d r ; \frac{1}{\gamma_{0}-1}} \\
& \quad \leq I_{1}(t, \theta, x)+c_{f}^{\gamma_{0}-1} I_{2}(t, \theta, x)+c_{f}^{\gamma_{0}-1} I_{3}(t, \theta, x)+c_{f}^{\gamma_{0}-1} I_{4}(t, \theta, x) .
\end{aligned}
$$

Step 3: We will show that, for each $\theta \geq 0$ and $x \in E, I_{1}(t, \theta, x) \underset{t \rightarrow \infty}{\longrightarrow} 0$. Notice that, by (1.21) and since $\left\langle f, \phi^{*}\right\rangle_{m}=1$,

$$
\Pi_{x}^{(\phi)}\left[\left(\phi^{-1} f\right)\left(\xi_{t}\right)\right]=\phi(x)^{-1} \Pi_{x}\left[f\left(\xi_{t}\right) e^{-\int_{0}^{t} \beta\left(\xi_{s}\right) d s}\right]=\phi(x)^{-1} P_{t}^{\beta} f(x) \underset{t \rightarrow \infty}{\longrightarrow} 1, \quad x \in E .
$$

Therefore,

$$
\begin{aligned}
& e^{-J_{G}(r)}-\left\|\left(\phi^{-1} f\right)\left(\xi_{t}\right)^{\gamma_{0}-1} e^{-J_{G}(r)}\right\|_{\Pi_{x}^{(\phi)} ; \frac{1}{\gamma_{0}-1}} \\
& \quad=e^{-J_{G}(r)}\left(1-\Pi_{x}^{(\phi)}\left[\left(\phi^{-1} f\right)\left(\xi_{t}\right)\right]^{\gamma_{0}-1}\right) \underset{t \rightarrow \infty}{\longrightarrow} 0, \quad x \in E, r \geq 0 .
\end{aligned}
$$

We also have the following bound:

$$
\left|e^{-J_{G}(r)}-\left\|\left(\phi^{-1} f\right)\left(\xi_{t}\right)^{\gamma_{0}-1} e^{-J_{G}(r)}\right\|_{\Pi_{x}^{(\phi)} ; \frac{1}{\gamma_{0}-1}}\right| \leq 1+c_{f}^{\gamma_{0}-1} .
$$

Therefore, by the bounded convergence theorem, we have that, for each $\theta \geq 0$ and $x \in E$, $I_{1}(t, \theta, x) \underset{t \rightarrow \infty}{\longrightarrow} 0$.

Step 4: We will show that, for each $\theta \geq 0$ and $x \in E, I_{2}(t, \theta, x) \underset{t \rightarrow \infty}{\longrightarrow} 0$. Notice that, according to (3.27) and (3.30), for each $t \geq 0$ and $r \geq 0$,

$$
\begin{aligned}
& J_{G}(r)-J_{G}^{\prime}(t, r, \xi) \\
& \quad=\int_{0}^{1} \gamma_{0} G\left(r u^{\frac{1}{\gamma_{0}-1}}\right)^{\gamma_{0}-1}\left(1-\left(\gamma_{0}-1\right) \mathbf{1}_{\gamma(\cdot)=\gamma_{0}} \kappa \phi^{\gamma_{0}-1} t u \eta_{u t}^{\gamma_{0}-1}\right)\left(\xi_{(1-u) t}\right) \frac{d u}{u}
\end{aligned}
$$




$$
=\int_{0}^{1} \gamma_{0} G\left(r u^{\frac{1}{\gamma_{0}-1}}\right)^{\gamma_{0}-1}\left(1-C_{X}^{-1} \mathbf{1}_{\gamma(\cdot)=\gamma_{0}} \kappa \phi^{\gamma_{0}-1}\right)\left(\xi_{(1-u) t}\right) \frac{d u}{u} .
$$

Also notice that, according to (3.32), for each $r \geq 0, u \in[0,1]$ and $x \in E$,

$$
\begin{array}{rl}
\mid \gamma_{0} & G\left(r u^{\frac{1}{\gamma_{0}-1}}\right)^{\gamma_{0}-1}\left(1-C_{X}^{-1} \mathbf{1}_{\gamma(\cdot)=\gamma_{0}} \kappa \phi^{\gamma_{0}-1}\right)(x) \frac{1}{u} \mid \\
\leq & \frac{\gamma_{0}}{u} G\left(r u^{\frac{1}{\gamma_{0}-1}}\right)^{\gamma_{0}-1}\left|\left(1-C_{X}^{-1} \mathbf{1}_{\gamma(\cdot)=\gamma_{0}} \kappa \phi^{\gamma_{0}-1}\right)(x)\right| \\
\leq \gamma_{0} r^{\gamma_{0}-1}\left(1+\left\|C_{X}^{-1} \mathbf{1}_{\gamma(\cdot)=\gamma_{0}} \kappa \phi^{\gamma_{0}-1}\right\|_{\infty}\right) .
\end{array}
$$

Therefore, according to Lemma 2.11 and the definition of $C_{X}$, we have that, for each $r \geq 0$ and $x \in E$,

$$
J_{G}(r)-J_{G}^{\prime}(t, r, \xi) \underset{t \rightarrow \infty}{\stackrel{L^{2}\left(\Pi_{x}^{(\phi)}\right)}{\longrightarrow}} \int_{0}^{1} \frac{\gamma_{0}}{u} G\left(r u^{\frac{1}{\gamma_{0}-1}}\right)^{\gamma_{0}-1}\left\langle 1-C_{X}^{-1} \mathbf{1}_{\gamma(\cdot)=\gamma_{0}} \kappa \phi^{\gamma_{0}-1}, \phi \phi^{*}\right\rangle_{m} d u=0 .
$$

According to (3.33) and (3.35), we have that, for each $r \geq 0$ and $t \geq 0$,

$$
\left|J_{G}(r)-J_{G}^{\prime}(t, r, \xi)\right| \leq\left(\gamma_{0}+c_{4}\right) r^{\gamma_{0}-1} .
$$

Therefore, according to the bounded convergence theorem, we have that, for each $r \geq 0$ and $x \in E$,

$$
\left\|J_{G}(r)-J_{G}^{\prime}(t, r, \xi)\right\|_{\Pi_{x}^{(\phi)} ; \frac{1}{\gamma_{0}-1}} \underset{t \rightarrow \infty}{\longrightarrow} 0 .
$$

According to (3.36), we have that, for each $\theta \geq 0, r \in[0, \theta]$ and $x \in E$,

$$
\left\|J_{G}(r)-J_{G}^{\prime}(t, r, \xi)\right\|_{\Pi_{x}^{(\phi)} ; \frac{1}{\gamma_{0}-1}} \leq\left(\gamma_{0}+c_{4}\right) \theta^{\gamma_{0}-1} .
$$

Finally, according to the bounded convergence theorem, we have that, for each $\theta \geq 0$ and $x \in E, I_{2}(t, \theta, x) \underset{t \rightarrow \infty}{\longrightarrow} 0$.

Step 5: We will show that, for each $\theta \geq 0$ and $x \in E, I_{4}(t, \theta, x) \underset{t \rightarrow \infty}{\longrightarrow} 0$. We first note that, for each $t \geq 0$ and $r \geq 0$, we have

$$
J_{g}(t, r, \xi)-J_{g}^{\prime}(t, r, \xi)=\left(\gamma_{0}-1\right) t \int_{0}^{1}\left(\mathbf{1}_{\gamma(\cdot)>\gamma_{0}} \kappa \gamma \cdot\left(\phi \eta_{u t}\right)^{\gamma-1} g\left(u t, r u^{\frac{1}{\gamma_{0}-1}}, \cdot\right)^{\gamma-1}\right)\left(\xi_{(1-u) t}\right) d u .
$$

We then note that, according (3.34) and the definition of $\eta_{t}$, for each $r \geq 0, u \in(0,1)$ and $x \in E$, we have

$$
\begin{aligned}
\left(\gamma_{0}\right. & -1) t \mathbf{1}_{\gamma(x)>\gamma_{0}} \kappa(x) \gamma(x)\left(\phi(x) \eta_{u t}\right)^{\gamma(x)-1} g\left(u t, r u^{\frac{1}{\gamma_{0}-1}}, x\right)^{\gamma(x)-1} \\
& \leq\left(\gamma_{0}-1\right)\left\|\kappa \gamma \cdot\left(c_{f} r \phi\right)^{\gamma-1}\right\|_{\infty} \mathbf{1}_{\gamma(x)>\gamma_{0}} t \eta_{u t}^{\gamma(x)-1} u^{\frac{\gamma(x)-1}{\gamma_{0}-1}} \\
& =\left(\gamma_{0}-1\right)\left\|\kappa \gamma \cdot\left(c_{f} r \phi\right)^{\gamma-1}\right\|_{\infty} \mathbf{1}_{\gamma(x)>\gamma_{0}} t\left(C_{X}\left(\gamma_{0}-1\right) u t\right)^{-\frac{\gamma(x)-1}{\gamma_{0}-1}} u^{\frac{\gamma(x)-1}{\gamma_{0}-1}} \\
& \leq\left(\gamma_{0}-1\right) \mathbf{1}_{\gamma(x)>\gamma_{0}} t^{1-\frac{\gamma(x)-1}{\gamma_{0}-1}}\left\|\kappa \gamma \cdot\left(c_{f} r \phi\right)^{\gamma-1}\right\|_{\infty} \sup _{x \in E}\left(C_{X}\left(\gamma_{0}-1\right)\right)^{-\frac{\gamma(x)-1}{\gamma_{0}-1}} \\
& \underset{t \rightarrow \infty}{\longrightarrow} 0 .
\end{aligned}
$$


This also gives an upper bound: For each $r \geq 0, u \in(0,1), x \in E$ and $t \geq 1$, we have

$$
\begin{gathered}
\left(\gamma_{0}-1\right) t \mathbf{1}_{\gamma(x)>\gamma_{0}} \kappa(x) \gamma(x)\left(\phi(x) \eta_{u t}\right)^{\gamma(x)-1} g\left(u t, r u^{\frac{1}{\gamma_{0}-1}}, x\right)^{\gamma(x)-1} \\
\leq\left(\gamma_{0}-1\right)\left\|\kappa \gamma \cdot\left(c_{f} r \phi\right)^{\gamma-1}\right\|_{\infty} \sup _{x \in E}\left(C_{X}\left(\gamma_{0}-1\right)\right)^{-\frac{\gamma(x)-1}{\gamma_{0}-1}} .
\end{gathered}
$$

Now, with (3.37), (3.38) and (3.40), we can apply Lemma 2.11 to the function

$$
(y, u, t) \mapsto\left(\gamma_{0}-1\right) t \mathbf{1}_{\gamma(y)>\gamma_{0}} \kappa(y) \gamma(y)\left(\phi(y) \eta_{u t}\right)^{\gamma(y)-1} g\left(u t, r u^{\frac{1}{\gamma_{0}-1}}, y\right)^{\gamma(y)-1},
$$

which says that, for each $r \geq 0$,

$$
J_{g}(t, r, \xi)-J_{g}^{\prime}(t, r, \xi) \underset{t \rightarrow \infty}{\stackrel{L^{2}\left(\Pi_{x}^{(\phi)}\right)}{\longrightarrow}} 0 .
$$

According to (3.37) and (3.39), for each $r \geq 0$ and $t \geq 1$, we have that

$$
\left|J_{g}(t, r, \xi)-J_{g}^{\prime}(t, r, \xi)\right| \leq\left(\gamma_{0}-1\right)\left\|\kappa \gamma \cdot\left(c_{f} r \phi\right)^{\gamma-1}\right\|_{\infty} \sup _{x \in E}\left(C_{X}\left(\gamma_{0}-1\right)\right)^{-\frac{\gamma(x)-1}{\gamma_{0}-1}} .
$$

Therefore, according to the bounded convergence theorem, for each $r \geq 0$ and $x \in E$, we have that

$$
\left\|J_{g}^{\prime}(t, r, \xi)-J_{g}(t, r, \xi)\right\|_{\Pi_{x}^{(\phi)} ; \frac{1}{\gamma_{0}-1}} \underset{t \rightarrow \infty}{\longrightarrow} 0 .
$$

According to (3.40), for each $\theta \geq 0, r \in[0, \theta], t \geq 1$ and $x \in E$, we have that

$$
\left\|J_{g}^{\prime}(t, r, \xi)-J_{g}(t, r, \xi)\right\|_{\Pi_{x}^{(\phi)} ; \frac{1}{\gamma_{0}-1}} \leq\left(\gamma_{0}-1\right)\left\|\kappa \gamma \cdot\left(c_{f} \theta \phi\right)^{\gamma-1}\right\|_{\infty} \sup _{x \in E}\left(C_{X}\left(\gamma_{0}-1\right)\right)^{-\frac{\gamma(x)-1}{\gamma_{0}-1}} .
$$

Therefore, according to the bounded convergence theorem, for each $\theta \geq 0$ and $x \in E$, we have that $I_{4}(t, \theta, x) \underset{t \rightarrow \infty}{\longrightarrow} 0$.

Step 6: We will show that

$$
\limsup _{t \rightarrow \infty} I_{3}(t, \theta, x) \leq \gamma_{0}\left(\int_{0}^{\theta}\left\|M\left(r u^{\frac{1}{\gamma_{0}-1}}\right)\right\|_{\mathbf{1}_{0 \leq u \leq 1} \frac{d u}{u} ; \gamma_{0}-1} d r\right)^{\gamma_{0}-1}, \quad \theta \geq 0, x \in E,
$$

where

$$
M(t, r, x):=\left|G(r)^{\gamma_{0}-1}-g(t, r, x)^{\gamma_{0}-1}\right|^{\frac{1}{\gamma_{0}-1}}, \quad t \geq 0, r \geq 0, x \in E,
$$

and

$$
M(r, x):=\limsup _{t \rightarrow \infty} M(t, r, x) ; \quad M(r):=\sup _{x \in E} M(r, x), \quad r \geq 0, x \in E .
$$

Notice that, according to (3.32) and (3.34), we have the following bound:

$$
M(t, r, x) \leq\left|r^{\gamma_{0}-1}+c_{f}^{\gamma_{0}-1} r^{\gamma_{0}-1}\right|^{\frac{1}{\gamma_{0}-1}}=: c_{6} r,
$$

where the constant $c_{6}$ is independent of $t$ and $x$. Therefore, we have

$$
M(r, x) \leq M(r) \leq c_{6} r, \quad r \geq 0, x \in E .
$$

From the definition of $J_{G}^{\prime}, J_{g}^{\prime}$ and $\eta_{t}$, we have for each $t \geq 0$ and $r \geq 0$,

$$
\left|J_{G}^{\prime}(t, r, \xi)-J_{g}^{\prime}(t, r, \xi)\right|
$$




$$
\begin{aligned}
& \leq \gamma_{0}\left(\gamma_{0}-1\right) t \int_{0}^{1}\left(\mathbf{1}_{\gamma(\cdot)=\gamma_{0}} \kappa \cdot\left(\phi \eta_{u t}\right)^{\gamma_{0}-1} M\left(u t, r u^{\frac{1}{\gamma_{0}-1}}, \cdot\right)^{\gamma_{0}-1}\right)\left(\xi_{(1-u) t}\right) d u \\
& =\gamma_{0} C_{X}^{-1} \int_{0}^{1}\left(\mathbf{1}_{\gamma(\cdot)=\gamma_{0}} \kappa \phi^{\gamma_{0}-1} u^{-1} M\left(u t, r u^{\frac{1}{\gamma_{0}-1}}, \cdot\right)^{\gamma_{0}-1}\right)\left(\xi_{(1-u) t}\right) d u
\end{aligned}
$$

According to (3.41), we have the following upper bound:

$$
u^{-1} M\left(u t, r u^{\frac{1}{\gamma_{0}-1}}, x\right) \leq c_{6} r u^{\frac{2-\gamma_{0}}{\gamma_{0}-1}} \leq c_{6} r, \quad u \in(0,1), r \geq 0, t \geq 0, x \in E .
$$

Therefore, fixing an $r \geq 0$, we can apply Lemma 2.12 to the function

$$
(y, u, t) \mapsto \gamma_{0} C_{X}^{-1} \mathbf{1}_{\gamma(y)=\gamma_{0}} \kappa(y) \phi(y)^{\gamma_{0}-1} u^{-1} M\left(u t, r u^{\frac{1}{\gamma_{0}-1}}, y\right)^{\gamma_{0}-1}
$$

since it is a bounded Borel function on $E \times(0,1) \times[0, \infty)$. Now, according to Lemma 2.12, (3.42) and the definitions of $M(r, x), M(r)$ and $C_{X}$, we have

$$
\begin{aligned}
& \limsup _{t \rightarrow \infty}\left\|J_{G}^{\prime}(t, r, \xi)-J_{g}^{\prime}(t, r, \xi)\right\|_{\Pi_{x}^{\phi} ; \frac{1}{\gamma_{0}-1}} \\
& \quad \leq \gamma_{0} C_{X}^{-1} \int_{0}^{1}\left\langle\mathbf{1}_{\gamma(\cdot)=\gamma_{0}} \kappa \phi^{\gamma_{0}-1} M\left(r u^{\frac{1}{\gamma_{0}-1}}, \cdot\right)^{\gamma_{0}-1}, \phi \phi^{*}\right\rangle_{m} \frac{d u}{u} \\
& \quad \leq \gamma_{0} \int_{0}^{1} M\left(r u^{\frac{1}{\gamma_{0}-1}}\right)^{\gamma_{0}-1} \frac{d u}{u} .
\end{aligned}
$$

We recall the reverse Fatou's lemma in $L^{p}$ with $p \geq 1$ : Let $\left(f_{n}\right)_{n \in \mathbb{N}}$ be a sequence of non-negative measurable functions defined on a measure space $S$ with $\sigma$-finite measure $\mu$. If there exists a non-negative $L^{p}(\mu)$-integrable function $g$ on $S$ such that $f_{n} \leq g$ for all $n$, then according to the classical reverse Fatou's lemma, we have

$$
\limsup _{n \rightarrow \infty}\left\|f_{n}\right\|_{\mu ; p}=\left(\limsup _{n \rightarrow \infty} \int f_{n}^{p} d \mu\right)^{\frac{1}{p}} \leq\left(\int \limsup _{n \rightarrow \infty} f_{n}^{p} d \mu\right)^{\frac{1}{p}}=\left\|\limsup _{n \rightarrow \infty} f_{n}\right\|_{\mu ; p} .
$$

Using this version of the reverse Fatou's lemma and (3.43), we get that

$$
\begin{aligned}
& \limsup _{t \rightarrow \infty} I_{3}(t, \theta, x) \leq\left\|\limsup _{t \rightarrow \infty}\right\| J_{G}^{\prime}(t, r, \xi)-J_{g}^{\prime}(t, r, \xi)\left\|_{\Pi_{x}^{(\phi)} ; \frac{1}{\gamma_{0}-1}}\right\|_{\mathbf{1}_{0 \leq r \leq \theta} d r ; \frac{1}{\gamma_{0}-1}} \\
& \quad \leq\left\|\gamma_{0} \int_{0}^{1} M\left(r u^{\frac{1}{\gamma_{0}-1}}\right)^{\gamma_{0}-1} \frac{d u}{u}\right\|_{\mathbf{1}_{0 \leq r \leq \theta} d r ; \frac{1}{\gamma_{0}-1}} \\
& \quad=\gamma_{0}\left(\int_{0}^{\theta}\left(\int_{0}^{1} M\left(r u^{\frac{1}{\gamma_{0}-1}}\right)^{\gamma_{0}-1} \frac{d u}{u}\right)^{\frac{1}{\gamma_{0}-1}} d r\right)^{\gamma_{0}-1} \\
& \quad=\gamma_{0}\left(\int_{0}^{\theta}\left\|M\left(r u^{\frac{1}{\gamma_{0}-1}}\right)\right\|_{\mathbf{1}_{0 \leq u \leq 1} \frac{d u}{u} ; \gamma_{0}-1} d r\right)^{\gamma_{0}-1}, \quad \theta \geq 0, x \in E .
\end{aligned}
$$

Step 7: We will show that $M(\theta)=0$ for each $\theta \geq 0$. We first claim that

$$
M(\theta) \leq c_{M} \int_{0}^{\theta}\left\|M\left(r u^{\frac{1}{\gamma_{0}-1}}\right)\right\|_{\mathbf{1}_{0 \leq u \leq 1} \frac{d u}{u} ; \gamma_{0}-1} d r, \quad \theta \geq 0
$$


for some constant $c_{M}>0$. In fact, a direct application of Steps 2-6 gives that, for each $t \geq 0$ and $x \in E$ :

$$
\begin{aligned}
& M(r, x)^{\gamma_{0}-1}=\limsup _{t \rightarrow \infty} M(t, r, x)^{\gamma_{0}-1}=\limsup _{t \rightarrow \infty}\left|G(r)^{\gamma_{0}-1}-g(t, r, x)^{\gamma_{0}-1}\right| \\
& \quad \leq \limsup _{t \rightarrow \infty}\left(I_{1}(t, \theta, x)+c_{f}^{\gamma_{0}-1} I_{2}(t, \theta, x)+c_{f}^{\gamma_{0}-1} I_{3}(t, \theta, x)+c_{f}^{\gamma_{0}-1} I_{4}(t, \theta, x)\right) \\
& \quad=c_{f}^{\gamma_{0}-1} \limsup _{t \rightarrow \infty} I_{3}(t, \theta, x) \leq c_{f}^{\gamma_{0}-1} \gamma_{0}\left(\int_{0}^{\theta}\left\|M\left(r u^{\frac{1}{\gamma_{0}-1}}\right)\right\|_{\mathbf{1}_{0 \leq u \leq 1} \frac{d u}{u} ; \gamma_{0}-1} d r\right)^{\gamma_{0}-1} .
\end{aligned}
$$

Therefore, for each $\theta \geq 0$,

$$
M(\theta)=\sup _{x \in E} M(r, x) \leq c_{f} \gamma_{0}^{\frac{1}{\gamma_{0}-1}} \int_{0}^{\theta}\left\|M\left(r u^{\frac{1}{\gamma_{0}-1}}\right)\right\|_{\mathbf{1}_{0 \leq u \leq 1} \frac{d u}{u} ; \gamma_{0}-1} d r .
$$

According to that $M(\theta) \leq c_{6} \theta$ for each $\theta$, we can apply Lemma A.8 to the above inequality to get the desired result.

Step 8: Finally, $M \equiv 0$ clearly implies that $\lim _{t \rightarrow \infty} I_{3}(t, \theta, x)=0$, and thus completes the verification of (3.25).

\section{Appendix A.}

A.1. Examples. In this Subsection, we briefly recall from [26] some examples of Markov processes satisfying Assumptions 1 and 3. We will not try to give the most general examples. For details and more examples, we refer our readers to [26].

Example A.1. Suppose that $E$ is a finite state space and $m$ is the counting measure on $E$. Let $\xi$ be an irreducible, continuous-time Markov chain. Then the semigroup $\left(P_{t}\right)_{t \geq 0}$ of $\xi$ satisfies Assumptions 1 and 3 .

Example A.2. Suppose that $E$ is a bounded Lipschitz connected open set of $\mathbb{R}^{d}$ and that $m$ denotes the Lebesgue measure on $E$. Let $\xi$ be the subprocess in $E$ of a diffusion process in $\mathbb{R}^{d}$ corresponding to a uniformly elliptic divergence form second order differential operator. Then the semigroup $\left(P_{t}\right)_{t \geq 0}$ of $\xi$ satisfies Assumptions 1 and 3 .

Example A.3. Suppose that $E$ is the closure of a bounded $C^{2}$ connected open set of $\mathbb{R}^{d}$ and that $m$ denotes the Lebesgue measure on $E$. Let $\xi$ be the reflecting Brownian motion in $E$. Then the semigroup $\left(P_{t}\right)_{t \geq 0}$ of $\xi$ satisfies Assumptions 1 and 3 .

Example A.4. Suppose that $E$ is a bounded open set of $\mathbb{R}^{d}$ and $m$ denotes the Lebesgue measure on $E$. $\xi$ be the subprocesses in $E$ of any of the subordinate Brownian motions studied in [15, 16]. Then the semigroup $\left(P_{t}\right)_{t \geq 0}$ of $\xi$ satisfies Assumptions 1 and 3.

Example A.5. Suppose $a>2$ is a constant. Assume that $E=\mathbb{R}^{d}$ and $m$ is the Lebesgue measure on $\mathbb{R}^{d}$. Let $\xi$ be a Markov process on $\mathbb{R}^{d}$ corresponding to the infinitesimal generator $\Delta-|x|^{a}$. Then the semigroup $\left(P_{t}\right)_{t \geq 0}$ of $\xi$ satisfies Assumption 1 and 3 ,

Example A.6. Assume that $E=\mathbb{R}^{d}$ and $m$ is the Lebesgue measure on $\mathbb{R}^{d}$. Suppose that $V$ is a nonnegative and locally bounded function on $\mathbb{R}^{d}$ such that there exist $R>0$ 
and $M \geq 1$ such that for all $|x|>R$,

$$
M^{-1}(1+V(x)) \leq V(y) \leq M(1+V(x)), \quad y \in B(x, 1),
$$

and that

$$
\lim _{|x| \rightarrow \infty} \frac{V(x)}{\log |x|}=\infty
$$

Suppose $\beta \in(0,2)$ is a constant. Let $\xi$ be a Markov process on $\mathbb{R}^{d}$ corresponding to the infinitesimal generator $-(-\Delta)^{\beta / 2}-V(x)$. Then the semigroup $\left(P_{t}\right)_{t \geq 0}$ of $\xi$ satisfies Assumptions 1 and 3 .

Example A.7. Suppose that $\beta \in(0,2)$ and that $\xi^{(1)}=\left\{\xi_{t}^{(1)}: t \geq 0\right\}$ is a strictly $\beta$-stable process in $\mathbb{R}^{d}$. Suppose that, in the case $d \geq 2$, the spherical part $\eta$ of the Lévy measure $\mu$ of $\xi^{(1)}$ satisfies the following assumption: there exist a positive function $\Phi$ on the unit sphere $S$ in $\mathbb{R}^{d}$ and $\kappa>1$ such that

$$
\Phi=\frac{d \eta}{d \sigma} \quad \text { and } \quad \kappa^{-1} \leq \Phi(z) \leq \kappa \quad \text { on } S
$$

where $\sigma$ is the surface measure on $S$. In the case $d=1$, we assume that the Lévy measure of $\xi^{(1)}$ is given by

$$
\mu(d x)=c_{1} x^{-1-\beta} 1_{\{x>0\}}+c_{2}|x|^{-1-\beta} 1_{\{x<0\}}
$$

with $c_{1}, c_{2}>0$. Suppose that $E$ is a bounded open set in $\mathbb{R}^{d}$ and $m$ is the Lebesgue measure on $E$. Let $\xi$ be the process in $E$ obtained by killing $\xi^{(1)}$ upon exiting $E$. Then the semigroup $\left(P_{t}\right)_{t \geq 0}$ of $\xi$ satisfies Assumptions 1 and 3 ,

A.2. Analytical results. In this Subsection, we give the proofs of the two lemmas used in the proof of Theorem 1.1(3). We think these two lemmas are of independent interest.

We first recall the following notation: If $f$ is a measurable function which is $L^{p}$ integrable on the measure space $(S, \mathscr{S}, \mu)$ with $p>0$, then we write

$$
\|f\|_{\mu ; p}:=\left(\int_{S}|f|^{p} d \mu\right)^{\frac{1}{p}}
$$

Notice that, when $p \geq 1,\|f\|_{\mu ; p}$ is simply the $L^{p}$ norm of $f$ with respect to the measure $\mu$. However, when $p \in(0,1),\|\cdot\|_{\mu ; p}$ is not a norm.

Lemma A.8. Suppose that $\alpha \in(1,2)$. Suppose that $F$ is a non-negative function on $[0, \infty)$ satisfying the property that there exists a constant $C>0$ such that $F(\theta) \leq C \theta$ for all $\theta \geq 0$ and

$$
F(\theta) \leq C \int_{0}^{\theta}\left\|F\left(r u^{\frac{1}{\alpha-1}}\right)\right\|_{\mathbf{1}_{0<u<1} \frac{d u}{u} ; \alpha-1} d r, \quad \theta \geq 0 .
$$

Then $F \equiv 0$.

Proof. We claim that for each $k \in \mathbb{N}$, we have

$$
F(\theta) \leq \frac{C^{k} \theta^{k}}{k !}, \quad \theta \geq 0 .
$$


In fact, when $k=1$ this is trival. Now if $(\underline{A .2})$ is true for a fixed $k \in \mathbb{N}$, then from

$$
\begin{aligned}
& F(\theta) \leq C \int_{0}^{\theta}\left\|F\left(r u^{\frac{1}{\alpha-1}}\right)\right\|_{\mathbf{1}_{0<u<1} \frac{d u}{u} ; \alpha-1} d r \leq C \int_{0}^{\theta}\left\|\frac{1}{k !}\left(C r u^{\frac{1}{\alpha-1}}\right)^{k}\right\|_{\mathbf{1}_{0<u<1} \frac{d u}{u} ; \alpha-1} d r \\
& \leq \frac{C^{k+1}}{k !}\left(\int_{0}^{\theta} r^{k} d r\right) \cdot\left\|u^{\frac{k}{\alpha-1}}\right\|_{\mathbf{1}_{0<u<1} \frac{d u}{u} ; \alpha-1} \leq \frac{C^{k+1} \theta^{k+1}}{(k+1) !}
\end{aligned}
$$

we have that $(\underline{A .2})$ is true for $k+1$. Therefore, by induction, (A.2) is true for all $k \in \mathbb{N}$.

Letting $k \rightarrow \infty$ in (A.2), we get that $F(\theta)=0$ for each $\theta \geq 0$.

Lemma A.9. Suppose that $\alpha \in(1,2)$. The non-linear delay equation

$$
G(\theta)=\int_{0}^{\theta} \exp \left\{-\frac{\alpha}{\alpha-1} \int_{0}^{1} G\left(r u^{\frac{1}{\alpha-1}}\right)^{\alpha-1} \frac{d u}{u}\right\} d r, \quad \theta \geq 0,
$$

has a unique solution:

$$
G(\theta)=\left(\frac{1}{1+\theta^{-(\alpha-1)}}\right)^{\frac{1}{\alpha-1}}, \quad \theta \geq 0 .
$$

Proof. We first verify that $(\underline{A .4})$ is a solution of $(\underline{A .3})$. In fact, if $G(\theta)=\left(\frac{1}{1+\theta^{-(\alpha-1)}}\right)^{\frac{1}{\alpha-1}}$, then

$$
\begin{aligned}
\int_{0}^{\theta} & \exp \left\{-\frac{\alpha}{\alpha-1} \int_{0}^{1} G\left(r u^{\frac{1}{\alpha-1}}\right)^{\alpha-1} \frac{d u}{u}\right\} d r \\
& =\int_{0}^{\theta} \exp \left\{-\frac{\alpha}{\alpha-1} \int_{0}^{1} \frac{d u}{u+r^{-(\alpha-1)}}\right\} d r=\int_{0}^{\theta} \exp \left\{-\frac{\alpha}{\alpha-1} \log \frac{1+r^{-(\alpha-1)}}{r^{-(\alpha-1)}}\right\} d r \\
& =\int_{0}^{\theta}\left(\frac{1+r^{-(\alpha-1)}}{r^{-(\alpha-1)}}\right)^{-\frac{\alpha}{\alpha-1}} d r=\int_{0}^{\theta}\left(1+r^{-(\alpha-1)}\right)^{-\frac{\alpha}{\alpha-1}} r^{-\alpha} d r=G(\theta) .
\end{aligned}
$$

The last equality is due to $G(0)=0$ and

$$
\begin{aligned}
\frac{d}{d \theta} G(\theta) & =-\frac{1}{\alpha-1}\left(1+\theta^{-(\alpha-1)}\right)^{-\frac{1}{\alpha-1}-1} \frac{d}{d \theta} \theta^{-(\alpha-1)} \\
& =\left(1+\theta^{-(\alpha-1)}\right)^{-\frac{\alpha}{\alpha-1}} \theta^{-\alpha} .
\end{aligned}
$$

Now assume that $G_{0}$ is another solution to the equation (A.3), we then only have to show that $G_{0}=G$. This can be done by showing that $F(\theta)=0$ where

$$
F(\theta):=\left|G(\theta)^{\alpha-1}-G_{0}(\theta)^{\alpha-1}\right|^{\frac{1}{\alpha-1}}, \quad \theta \geq 0 .
$$

We claim that the non-negative function $F$ satisfies the inequality (A.1) with $C=$ $\alpha^{1 /(\alpha-1)}$. In fact, by the $L^{p}$ Minkowski inequality with $p=\frac{1}{\alpha-1}>1$, we have

$$
\begin{aligned}
& \left|G(\theta)^{\alpha-1}-G_{0}(\theta)^{\alpha-1}\right| \\
& \quad=\left|\left\|e^{-\alpha \int_{0}^{1} G\left(r u^{\frac{1}{\alpha-1}}\right)^{\alpha-1} \frac{d u}{u}}\right\|_{\mathbf{1}_{0<r<\theta} d r ; \frac{1}{\alpha-1}}-\left\|e^{-\alpha \int_{0}^{1} G_{0}\left(r u^{\frac{1}{\alpha-1}}\right)^{\alpha-1} \frac{d u}{u}}\right\|_{\mathbf{1}_{0<r<\theta} d r ; \frac{1}{\alpha-1}}\right| \\
& \quad \leq\left\|e^{-\alpha \int_{0}^{1} G\left(r u^{\frac{1}{\alpha-1}}\right)^{\alpha-1} \frac{d u}{u}}-e^{-\alpha \int_{0}^{1} G_{0}\left(r u^{\frac{1}{\alpha-1}}\right)^{\alpha-1} \frac{d u}{u}}\right\|_{\mathbf{1}_{0<r<\theta} d r ; \frac{1}{\alpha-1}}
\end{aligned}
$$




$$
\begin{aligned}
& \leq\left\|\alpha \int_{0}^{1} G\left(r u^{\frac{1}{\alpha-1}}\right)^{\alpha-1} \frac{d u}{u}-\alpha \int_{0}^{1} G_{0}\left(r u^{\frac{1}{\alpha-1}}\right)^{\alpha-1} \frac{d u}{u}\right\|_{1_{0<r<\theta} d r ; \frac{1}{\alpha-1}} \\
& \leq \alpha\left(\int_{0}^{\theta}\left(\int_{0}^{1}\left|G\left(r u^{\frac{1}{\alpha-1}}\right)^{\alpha-1}-G_{0}\left(r u^{\frac{1}{\alpha-1}}\right)^{\alpha-1}\right| \frac{d u}{u}\right)^{\frac{1}{\alpha-1}} d r\right)^{\alpha-1} .
\end{aligned}
$$

This implies the claim.

On the other hand, according to (A.3), we have that $G(\theta) \leq \theta$ and $G_{0}(\theta) \leq \theta$. Therefore, we also have that there is a constant $C_{1}>0$ such that $F(\theta) \leq C_{1} \theta$. Therefore, according to Lemma A.8, we have $F \equiv 0$ as desired.

Acknowledgment: We thank the referees for helpful comments on the first version of this paper.

\section{REFERENCES}

[1] Asmussen, S. and Hering, H.: Branching processes. Progress in Probability and Statistics, 3. Birkhäuser Boston, Inc., Boston, MA, 1983.

[2] Athreya, K. and Ney, P.: Functionals of critical multitype branching processes. Ann. Probability 2 (1974), 339-343.

[3] Athreya, K. and Ney, P.: Branching processes. Die Grundlehren der mathematischen Wissenschaften, Band 196. Springer-Verlag, New York-Heidelberg, 1972.

[4] Bingham, N., Goldie, C. and Teugels J.: Regular variation. Encyclopedia of Mathematics and its Applications, 27. Cambridge University Press, Cambridge, 1989.

[5] Borovkov, K.: A method for the proof of limit theorems for branching processes. Teor. Veroyatnost. i Primenen. 33 (1988), no. 1, 115-123; translation in Theory Probab. Appl. 33 (1988), no. 1, 105-113.

[6] Eckhoff, M., Kyprianou, A. and Winkel, M.: Spines, skeletons and the strong law of large numbers for superdiffusions. Ann. Probab. 43 (2015), no. 5, 2545-2610.

[7] Engländer, J. and Kyprianou, A.: Local extinction versus local exponential growth for spatial branching processes. Ann. Probab. 32 (2004), no. 1A, 78-99.

[8] Evans, S. and Perkins, E.: Measure-valued Markov branching processes conditioned on nonextinction. Israel J. Math. 71 (1990), no. 3, 329-337.

[9] Goldstein, M. and Hoppe, F.: Critical multitype branching processes with infinite variance. J. Math. Anal. Appl. 65 (1978), no. 3, 675-686.

[10] Harris, T.: The theory of branching processes. Die Grundlehren der Mathematischen Wissenschaften, Bd. 119 Springer-Verlag, Berlin; Prentice-Hall, Inc., Englewood Cliffs, N.J. 1963.

[11] Iyer, G., Leger, N. and Pego, R.: Limit theorems for Smoluchowski dynamics associated with critical continuous-state branching processes. Ann. Appl. Probab. 25 (2015), no. 2, 675-713.

[12] Joffe, A. and Spitzer, F.: On multitype branching processes with $\rho \leq 1$. J. Math. Anal. Appl. 19 (1967), 409-430.

[13] Kesten, H., Ney, P. and Spitzer, F.: The Galton-Watson process with mean one and finite variance. Teor. Verojatnost. i Primenen. 11 (1966), 579-611.

[14] Kim, P. and Song, R.: Intrinsic ultracontractivity of non-symmetric diffusion semigroups in bounded domains. Tohoku Math. J. (2) 60 (2008), no. 4, 527-547.

[15] Kim, P., Song, R. and Vondracek, Z.: Two-sided Green function estimates for killed subordinate Brownian motions. Proc. London Math. Soc. 104 (2012), 927-958.

[16] Kim, P., Song, R. and Vondracek, Z.: Potential theory of subordinate Brownian motions with Gaussian components. Stoch. Proc. Appl. 123 (2013), 764-795.

[17] Kolmogorov, A.: Zur lösung einer biologischen aufgabe. Comm. Math. Mech. Chebyshev Univ. Tomsk 2 (1938), no. 1, 1-12. 
[18] Kyprianou, A.: Fluctuations of Lévy processes with applications. Introductory lectures. Second edition. Universitext. Springer, Heidelberg, 2014.

[19] Kyprianou, A. and Pardo, J.: Continuous-state branching processes and self-similarity. J. Appl. Probab. 45 (2008), no. 4, 1140-1160.

[20] Li, Z.: Measure-valued branching Markov processes. Probability and its Applications (New York), Springer, Heidelberg, 2011.

[21] Liu, R.-L., Ren, Y.-X. and Song, R. L $\log L$ criterion for a class of superdiffusions. J. Appl. Probab. 46 (2009), no. 2, 479-496.

[22] Pakes, A.: Critical Markov branching process limit theorems allowing infinite variance. Adv. in Appl. Probab. 42 (2010), no. 2, 460-488.

[23] Powell, E.: An invariance principle for branching diffusions in bounded domains. Probab. Theory Related Fields 173 (2019), no. 3-4, 999-1062.

[24] Ren, Y.-X., Song, R. and Sun, Z.: A 2-spine decomposition of the critical Galton-Watson tree and a probabilistic proof of Yaglom's theorem. Electron. Commun. Probab. 23 (2018), Paper No. 42, 12 pp.

[25] Ren, Y.-X., Song, R. and Sun, Z.: Spine decompositions and limit theorems for a class of critical superprocesses. Acta Appl. Math. (2019). https://doi.org/10.1007/s10440-019-00243-7

[26] Ren, Y.-X., Song, R. and Zhang, R.: Limit theorems for some critical superprocesses. Illinois J. Math. 59 (2015), no. 1, 235-276.

[27] Ren, Y.-X., Song, R. and Zhang, R.: Central limit theorems for supercritical branching nonsymmetric Markov processes. Ann. Probab. 45 (2017), no. 1, 564-623.

[28] Ren, Y.-X., Yang, T. and Zhao, G.: Conditional limit theorems for critical continuous-state branching processes. Sci. China Math. 57 (2014), no. 12, 2577-2588.

[29] Schaefer, H.: Banach lattices and positive operators. Die Grundlehren der mathematischen Wissenschaften, Band 215. Springer-Verlag, New York-Heidelberg, 1974.

[30] Slack, R.: A branching process with mean one and possibly infinite variance. Z. Wahrscheinlichkeitstheorie und Verw. Gebiete 9 (1968), 139-145.

[31] Slack, R.: Further notes on branching processes with mean 1. Z. Wahrscheinlichkeitstheorie und Verw. Gebiete 25 (1972/73), 31-38.

[32] Vatutin, V.: Limit theorems for critical multitype Markov branching processes with infinite second moments. Mat. Sb. (N.S.) 103(145) (1977), no. 2, 253-264, 319.

[33] Yaglom, A.: Certain limit theorems of the theory of branching random processes. Doklady Akad. Nauk SSSR (N.S.) 56 (1947). 795-798.

[34] Zolotarev, V.: More exact statements of several theorems in the theory of branching processes. Teor. Veroyatnost. i Primenen. 2 (1957), 256-266.

Yan-Xia Ren, School of Mathematical Sciences, Peking University, Beijing, P. R. CHINA, 100871

E-mail address: yxren@math.pku.edu.cn

Renming Song, Dept of Mathematics, University of Illinois at Urbana-Champaign, URBANA, IL, USA, 61801

E-mail address: rsong@illinois.edu

Zhenyao Sun, School of Mathematics and Statistics, Wuhan University, Wuhan, Hubei, P. R. China, 430072

E-mail address: zhenyao.sun@pku.edu.cn 